\title{
Variational Method for Image-Based Inference of Internal Stress in Epithelial Tissues
}

\author{
Nicholas Noll, ${ }^{1,2,3}$ Sebastian J. Streichan, ${ }^{1,4}$ and Boris I. Shraiman ${ }^{1,4}$ \\ ${ }^{1}$ Department of Physics, University of California Santa Barbara, Santa Barbara, California 93106, USA \\ ${ }^{2}$ Biozentrum, University of Basel, Basel 4056, Switzerland \\ ${ }^{3}$ Swiss Institute of Bioinformatics, Lausanne 1015, Switzerland \\ ${ }^{4}$ Kavli Institute for Theoretical Physics, Santa Barbara, California 93106, USA
}

(Received 6 February 2019; revised manuscript received 10 September 2019; accepted 16 December 2019; published 26 March 2020)

\begin{abstract}
Cellular mechanics drives epithelial morphogenesis, the process wherein cells collectively rearrange to produce tissue-scale deformations that determine organismal shape. However, quantitative understanding of tissue mechanics is impaired by the difficulty of direct measurement of stress in vivo. This difficulty has spurred the development of image-based inference algorithms that estimate stress from snapshots of epithelial geometry. Such methods are challenged by sensitivity to measurement error and thus require accurate geometric segmentation for practical use. We overcome this difficulty by introducing a novel approach — the variational method of stress inference (VMSI) — which exploits the fundamental duality between stress and geometry at equilibrium of discrete mechanical networks that model confluent cellular layers. We approximate the apical geometry of an epithelial tissue by a 2D tiling with circular arc polygons in which arcs represent intercellular interfaces defined by the balance of local line tension and pressure differentials between adjacent cells. The mechanical equilibrium of such networks imposes extensive local constraints on circular arc polygon geometry. These constraints provide the foundation of VMSI which, starting with images of epithelial monolayers, simultaneously approximates both tissue geometry and internal forces, subject to the constraint of equilibrium. We find VMSI to be more robust than previous methods. Specifically, the VMSI performance is validated by the comparison of the predicted cellular and mesoscopic scale stress with the measured myosin II patterns during early Drosophila embryogenesis. VMSI prediction of a mesoscopic stress tensor correlates at the $80 \%$ level with the measured myosin distribution and reveals that most of the myosin activity in that case is involved in a static internal force balance within the epithelial layer. In addition to insight into cell mechanics, this study provides a practical method for nondestructive estimation of stress in live epithelial tissue.
\end{abstract}

DOI: $10.1103 /$ PhysRevX.10.011072

Subject Areas: Biological Physics, Interdisciplinary Physics, Mechanics

\section{INTRODUCTION}

Cell and tissue mechanics is an important factor that both affects and regulates animal and plant development and thus is a subject of active study in developmental biology and biophysics, reviewed extensively in Refs. [1-7]. Here we focus on the mechanics of animal epithelial cells that compose tissues in the form of two-dimensional monolayers with tight junctions between adjacent cells and adhesion (of the basal cellular surface) to the substrate extracellular matrix [8].

In the absence of a rigid substrate, mechanical properties of such monolayers are dominated by the tissue-wide network formed by cytoskeletal cortices coupled by

Published by the American Physical Society under the terms of the Creative Commons Attribution 4.0 International license. Further distribution of this work must maintain attribution to the author(s) and the published article's title, journal citation, and DOI. intercellular adherens junctions [9-12]. Cytoskeletal cortices are localized to the lateral sides of cells, just below the apical surface, and are made of actin fibers cross-linked by myosin II motors that actively generate tension within the cortex. The shape of cells within the tissue is determined by the balance of local actomyosin cytoskeletal contractility and the intracellular osmotic pressure [13], which acts to oppose the decrease in total cellular volume $[14,15]$. For the purpose of tissue-scale mechanics, the full threedimensional force balance that shapes individual cells can be approximated by an effective two-dimensional model of the apical cytoskeletal network. In this simplified $2 \mathrm{D}$ view, the contractility of the junctional actomyosin "belts" balances against an effective two-dimensional pressure that prevents the collapse of the apical area under cortical tension: this type of an approximation underlies the widely used "vertex model" approach to epithelial cell mechanics [1,16-18]. Vertex models, along with alternative models of epithelial mechanics, are reviewed in detail in Ref. [19]. 
Measuring mechanical properties of cells and tissues in vivo presents a considerable experimental challenge. AFM [20] and optical tweezer contact microscopy [21] have been used to probe the local rheology of individual cellular interfaces at great resolution but do not provide a direct readout of internal stress. The most common method for detecting stress in vivo is UV laser ablation, in which focused light "cuts" cytoskeletal bundles at the interface between adjacent cells and the resultant retraction velocity is used as a proxy for the local cortical tension [22]. Alternatively, UV laser ablation can be used to cut along a closed contour and measure resulting deformation of the enclosed 2D patch of cells, which provides an estimate of local stress in the tissue [23]. This method is convenient, as it does not require any special preparation of the sample; however, it is destructive and hence does not allow measurement of the global stress distribution across the tissue. Other methods use genetically encoded Förster resonance energy transfer tension sensors engineered into load carrying proteins [24] or employ measurements of deformation with implanted oil droplets [25,26]. These methods require specially prepared samples and are technically challenging, both in implementation and in quantitative interpretation. A comprehensive review of experimental mechanical measurement techniques may be found in Ref. [27].

The difficulty of direct experimental measurement of mechanical stress in developing tissues has stimulated alternative approaches that seek to leverage the availability of live imaging data [28-33]. For example, Refs. [29,31] introduced a method for inferring cellular stress from observed 2D cell geometry based on the assumption that the tissue is instantaneously in a mechanical equilibrium, described by a model parametrized directly by intercellular pressure and tension. In the simplest versions of the method [29,31], 2D geometry was parametrized by a polygonal tiling generally used in vertex models $[1,16]$. The validity of this approach, of course, rests upon the accuracy of the assumptions and approximations, which varies between tissues and conditions and has to be evaluated in each case; recent studies on force inference techniques show agreement [34]. The major challenge to the approach stems from the sensitivity to noise both in the measurement of cellular geometry as well as on the chosen prior distribution on the boundary conditions at the edge of the observed tissue domain. These difficulties have necessitated additional assumptions, introduced to regularize the inference problem [29,31,32]. A particularly direct generalization aims to extract additional information from the image data, most obviously provided by the observable curvature of cell interfaces [29,35]: an example of such an approach is the CellFIT toolkit [35]. Sensitivity to noise and the quality of the underlying image analysis, however, remains a major issue that impedes wide use of the method. Below, we develop a new approach, improving the image-based "mechanical inference" to the extent that makes it broadly applicable in the practice of experimental data analysis.

Specifically, in this paper we explore the general constraints imposed by mechanical equilibrium on the geometry of 2D cellular arrays that balance arbitrary interfacial tension against differential cellular pressure. Our analysis uncovers the mathematical duality between the geometry of the cell array and a triangulation formed by the equilibrium values of interfacial tensions [18], which in the general case of nonuniform pressures turns out to be nonplanar. This duality furnishes a set of highly nontrivial, local constraints on cellular geometry that can be used to simultaneously carry out image segmentation and infer internal forces. The stability of our mechanical inference method is achieved through a variational formulation of the inference problem. The power of this approach is twofold: (i) our representation of tissue geometry is constrained to be in mechanical equilibrium throughout the analysis and (ii) the inference is overdetermined and thus more robust with respect to image noise or information missing at the boundaries. As such, the constrained variational approach provides a stringent, quantitative assay for the compatibility of the network geometry with force balance.

Using synthetic data as a comparative benchmark, we show that our algorithm correctly infers mechanics under arbitrary pressure differentials and moderate measurement noise, performing better than existing methods. To illustrate its practical utility, we apply the algorithm to live imaging data from the early stages of Drosophila embryonic development and demonstrate its ability to accurately predict - based on cell geometry alone-the spatial distribution and anisotropy of myosin II, the molecular motor known as a generator of mechanical stress in the developing embryo [2,36-38]. Synthetic and real data tests suggest practical utility for the new mechanical inference algorithm as a tool for quantifying stress distribution in live tissue in the absence of direct measurement of local forces. The code used to segment apical cell morphology and to perform the variational inference can be found on Github [39].

\section{MECHANICAL EQUILIBRIUM OF 2D CELL ARRAYS WITH INTERNAL TENSION AND PRESSURE}

In order to model the mechanical state of the 2D epithelial tissue, we utilize a generalization of the standard vertex model $[16,29,35]$ which represents the epithelium by a planar polygonal tiling parametrized solely by the positions of vertices, the location where three or more cells meet, hereafter denoted by $\boldsymbol{r}_{i}$. We approximate the geometry of cells in the epithelial layer by a circular arc polygonal (CAP) tiling, as shown in Fig. 1(a), replacing the straight polygonal edges with circular arcs that correspond to tensed interfaces balancing pressure differentials between the adjacent cells as described by the YoungLaplace (YL) law $[35,40]$. The equilibrium geometry of a 

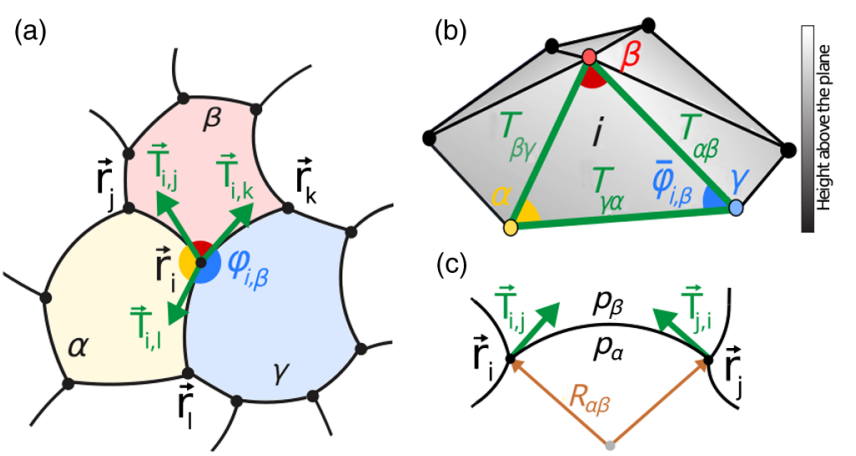

(c)

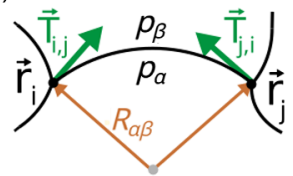

FIG. 1. Circular arc polygonal (CAP) tiling and its dual tension triangulation. (a) Circular arc polygons provide an approximate representation of the equilibrium geometry of a cell array, in which edges are represented as circular arcs and vertices are loci where three cells meet. $\phi_{j, \beta} \sim \pi$ implies that $T_{i j} \ll T_{i k}, T_{i l}$. (b) Force balance at vertex $i$ requires the three tension vectors tangent to each edge to sum up to zero, which defines a local triangle. As adjacent vertices in (a) share edges, the tension triangles of each vertex form a triangulated surface-the dual representation of force balance-with each triangular face corresponding to each vertex. Pressure differentials between cells result in a nonflat dual triangulation. The height above the $x y$ plane is represented as gray scale. (c) In mechanical equilibrium, the curvature of edge $\alpha \beta$ is controlled by pressure differences between adjacent cells, $p_{\alpha}-p_{\beta}$, and the interfacial tension $T_{\alpha \beta}$ by the Young-Laplace law [40].

CAP tiling is fully specified by the set of effective interfacial tensions $T_{\alpha \beta}$, where $\alpha, \beta$ label the cells partitioned by the given edge and the set of effective (2D) hydrostatic pressures $p_{\alpha}$ representing the contribution of bulk stress from the cell to the 2D apical force balance.

The dimensionality of the space of general CAP tilings is given by the number of internal degrees of freedom (d.o.f.): two positional d.o.f. for each vertex plus the radius of curvature for each edge. The total count is $2 v+e=7 c$, where $c, e, v$ respectively denote the number of cells, edges, and vertices within the tiling, which satisfy $v=2 c$ and $e=3 c$. (This count, which follows from Euler's formula [41], is exact on a torus and is approximate up to the boundary corrections, for a large planar array). Conversely, the mechanical state of the network is parametrized by $e+c=4 c$ parameters corresponding to independent interfacial tensions and cell pressures. Since the dimension of the space of all equilibrium CAP tilings, $4 c$, is lower than the dimension of general CAP tiling space, $7 c$, we conclude that mechanical equilibrium implies on average three constraints per cell. Our analysis below (i) defines a relation between the tiling geometry and tensions and pressures and (ii) uncovers the geometric constraints associated with mechanical balance. These constraints on CAP geometry serve as the foundation for our variational method of stress inference (VMSI).

Mechanical equilibrium of a CAP network is reached when tensions balance at every vertex and interfacial

curvature obeys the Young-Laplace law, which relates the radius of the circular arc forming interface $\alpha \beta, R_{\alpha \beta}$ to interfacial tension and the pressure differential across the interface: $R_{\alpha \beta}=T_{\alpha \beta} /\left[p_{\alpha}-p_{\beta}\right]$. Tension acts tangentially along network edges: Figure 1(a) depicts an example of force balance for vertex $i$ due to tensions of interfaces that connect it with adjacent vertices $j, k, l$. Specifically, tension $T_{i j}$ in the edge connecting vertices $i$ and $j$ acts on vertex $i$ with the force vector $\boldsymbol{T}_{i, j}$ acting along the $i j$ edge tangent at vertex $i$ (and pointing towards $j$ ). Similarly, we define the force vector $\boldsymbol{T}_{j, i}$ acting on vertex $j$. Importantly, these two vectors are not parallel, because CAP edges are generally curved; however, both have the same magnitude equal to the edge tension $\left|\boldsymbol{T}_{i, j}\right|=\left|\boldsymbol{T}_{j, i}\right|=T_{i j}$. We note that an edge can be labeled in two equivalent ways: either by the labels of cells the edge partitions or the vertices that the edge connects-e.g., edge $i j$ in Fig. 1(a) separates cells $\alpha, \beta$-so we shall define $T_{i j}=T_{\alpha \beta}$ and hereafter use both schemes interchangeably to simplify labeling in different contexts. Hence, force balance at vertex $i$ requires

$$
\boldsymbol{T}_{i, j}+\boldsymbol{T}_{i, k}+\boldsymbol{T}_{i, l}=0
$$

(with $j, k, l$ being vertices adjacent to $i$ ). Force balance can be reinterpreted geometrically as a triangle formed by the tension vectors incident on vertex $i$, with adjacent cells $\alpha, \beta$, $\gamma$, associated as the vertices of the "dual" tension triangle. Crucially, as the local force balance triangles associated with adjacent vertices share an edge, stitching together force balance conditions on all network vertices defines a tension triangulation dual to the CAP tiling. Dual triangulation vertices correspond to cells and triangular faces correspond to the vertices of the original CAP tiling. As discussed further in the Appendix A, curved edges impose angular "defects" between neighboring triangles, ultimately resulting in nonplanarity of the dual triangulation, depicted in Fig. 1(b).

We proceed to define the equilibrium constraints on CAP geometry and define the explicit relations between tensions, pressures, and geometric observables. By applying the sine law to an individual dual triangle [see Fig. 1(b)], one can relate the ratio of tensions in adjacent edges to the corresponding CAP angles, e.g., for the dual triangle $i$ (associated with adjacent cells $\alpha, \beta, \gamma$ ): $T_{\alpha \beta} / T_{\alpha \gamma}=\sin \varphi_{i, \gamma} / \sin \varphi_{i, \beta}$. Multiplying such ratios for a set of triangles that share a dual triangulation vertex-i.e., a cell-uncovers a nontrivial constraint on CAP tiling angles,

$$
\chi_{\alpha} \equiv \prod_{i \in V_{\alpha}} \frac{\sin \varphi_{i, \gamma}}{\sin \varphi_{i, \beta}}=\prod_{i \in V_{\alpha}} \frac{T_{\alpha \gamma}}{T_{\alpha \beta}}=1,
$$

where the product is taken over the set $\mathcal{V}_{\alpha}$ of vertices $i$ that belong to cell $\alpha$, while $\beta$ and $\gamma$ label other cells adjacent to $i$ in clockwise order, which means that each edge tension $T_{\alpha \beta}$ of the cell will appear exactly twice in the second product, 
once in the numerator, and once in the denominator, leading to the above equality to 1; see Fig. 1(b) for a depiction of the geometry. Equation (2) defines $c$ (one per cell) nontrivial constraints on the angles of an equilibrium CAP network which we recognize as the generalized form of the geometric compatibility condition introduced in Ref. [18]. Provided that the geometric constraints on the CAP tiling given by Eq. (2) are satisfied, the dual tension triangulation specifies all $T_{\alpha \beta}$ (up to an overall scale) - the key property making inference possible.

Given the set of tensions $T_{\alpha \beta}$ computable from the tension triangulation, pressures can be computed on the basis of the Young-Laplace law, depicted in Fig. 1(c), by solving a discrete Poisson equation on the dual triangulation:

$$
\sum_{\{\beta\}_{\alpha}}\left(p_{\alpha}-p_{\beta}\right)=\sum_{\{\beta\}_{\alpha}} \frac{T_{\alpha \beta}}{R_{\alpha \beta}} .
$$

Equation (3) represents $c$ equations (one for each $\alpha$ ) that define $c$ pressure unknowns up to the homogeneous solution which has to be fixed by the boundary conditions on $p_{\alpha}$.

Importantly, the YL law implies that $T_{\alpha \beta} / R_{\alpha \beta}+$ $T_{\beta \gamma} / R_{\beta \gamma}+T_{\gamma \alpha} / R_{\gamma \alpha}=0$ must be satisfied at each vertex (e.g., vertex $i$ shared by cells $\alpha, \beta, \gamma$ ). Using the sine law on the dual triangulation, this constraint is recast in the purely geometric form,

$$
\frac{\sin \left(\varphi_{i, \gamma}\right)}{R_{\alpha \beta}}+\frac{\sin \left(\varphi_{i, \alpha}\right)}{R_{\beta \gamma}}+\frac{\sin \left(\varphi_{i, \beta}\right)}{R_{\gamma \alpha}}=0,
$$

which defines $v=2 c$ (one per each vertex) geometric constraints that account for the difference in the number of pressure variables $c$ and the number of $R_{\alpha \beta}$ variables, $e=3 c$. Together, Eqs. (2) and (4) impose $3 c$ constraints on an equilibrium CAP network so that the dimensionality of the latter is indeed given by $4 c$, which is equal to the total number of tension and pressure variables. We note that mechanical equilibrium considerations discussed above cannot fix the overall scale of tensions and pressures and, as evident from Eq. (3), even with fixed tensions, pressure is defined only up to an additive constant.

\section{VARIATIONAL APPROACH TO IMAGE-BASED FORCE INFERENCE}

In principle, one can infer the underlying balanced mechanical state-the set of equilibrium interfacial tensions $\left\{T_{\alpha \beta}\right\}$ and pressures $\left\{p_{\alpha}\right\}$-by building the tension triangulation: utilizing local edge angles at each vertex to build each dual triangular face, and subsequently solving Eq. (3) to obtain cell pressures. This approach is contingent upon cell array geometry satisfying compatibility conditions defined by Eqs. (2) and (4). In practice it suffers from two major problems: (i) real cellular arrays undergoing morphogenesis are expected to be close to, but not exactly in, mechanical equilibrium, and (ii) the measurement of array geometry from imaging data will always be noisy and imperfect. As a result, an algorithm attempting to stitch together a global tension triangulation could rapidly accumulate errors that would dramatically impair resulting inference.

Recognizing that mechanical equilibrium cell array is at best an approximation to any observed epithelial tissue, it makes sense to approach the inference problem in the framework of least-squares fitting. Specifically, the idea is to approximate the observed cell array with the closest equilibrium CAP tiling. We begin by parametrizing the CAP tiling by circular arc centroids $\boldsymbol{\rho}_{\alpha \beta}$ (using from here on the bold face font to denote $2 \mathrm{D}$ vectors) and the associated radii of curvature $R_{\alpha \beta}$ and minimize the variational pseudoenergy function,

$$
E=\frac{1}{2 n_{e}} \sum_{(\alpha, \beta)} \sum_{n}^{N_{\alpha \beta}}\left(\left|\boldsymbol{r}_{\alpha \beta}(n)-\boldsymbol{\rho}_{\alpha \beta}\right|-R_{\alpha \beta}\right)^{2} .
$$

$\boldsymbol{r}_{\alpha \beta}(n)$ denotes the position of the $n$th pixel on edge $\alpha, \beta$ obtained directly from the segmentation and $N_{\alpha \beta}$ denotes the number of pixels segmented per edge. Equation (5) has a simple geometric interpretation-it penalizes the Euclidean distance between the estimated and measured circular arc for each pixel of a segmented edge. A graphical example of the fitting is shown in Fig. 2(a).

Arc centroids $\boldsymbol{\rho}_{\alpha \beta}$ and radii $R_{\alpha \beta}$ enter as $2 e+e=9 c$ variational parameters in Eq. (5). Yet, to form a CAP array,
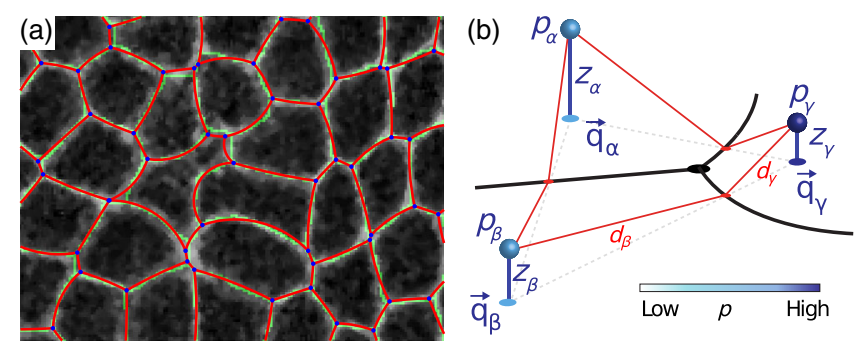

FIG. 2. Determination of equilibrium CAP tilings. (a) Fitting an equilibrium CAP tiling to imaging data. The best fit to raw data (shown in gray scale) by an equilibrium CAP tiling found by minimizing Eq. (5) is shown in red (with vertex positions in blue). The CAP contour is close, but distinct from the result of standard cell segmentation (see Sec. VII), here shown in green. (b) An example of vertex position $\boldsymbol{r}_{i}$ derived from the generalized Voronoi construction-i.e., generating points $\boldsymbol{q}_{\alpha}$ displaced to height $z_{\alpha}$ above the plane and a metric rescaling $p_{\alpha}$. Each CAP edge $\beta \gamma$ is the locus of points with a fixed ratio of distances to a corresponding pair of generating points-e.g., $\sqrt{p_{\beta}} d_{\beta}=\sqrt{p_{\gamma}} d_{\gamma}$ in the above image. The vertex is defined by the intersection of all three arcs. Parameters of this generalized Voronoi construction (including $p_{\alpha}$ ) provide a convenient set of reduced variables used in the minimization of Eq. (5). 
the three circles drawn from centroids corresponding to edges that meet at one vertex must all pass through the same point which defines that vertex. This implies $v$ constraints, reducing the number of d.o.f. to $9 c-v=7 c$, which is exactly the number necessary to describe a general CAP tiling. We, however, wish to constrain our variational approach further, to the subset of CAP tilings corresponding to mechanical equilibrium, which has to satisfy geometric constraints imposed by Eqs. (2) and (4).

To implement these constraints we express $\boldsymbol{\rho}_{\alpha \beta}$ and $R_{\alpha \beta}$ in terms of a smaller number of independent variables that explicitly restrict our variational search to the $4 c$-dimensional space of equilibrium CAP tilings. In Appendix B, we demonstrate that all constraints are explicitly solved by the following representation for arc centroids,

$$
\boldsymbol{\rho}_{\alpha \beta}=\frac{p_{\beta} \boldsymbol{q}_{\beta}-p_{\alpha} \boldsymbol{q}_{\alpha}}{p_{\beta}-p_{\alpha}}
$$

and the radii of curvature,

$$
R_{\alpha \beta}=\sqrt{\frac{p_{\alpha} p_{\beta}\left|\boldsymbol{q}_{\alpha}-\boldsymbol{q}_{\beta}\right|^{2}}{\left(p_{\alpha}-p_{\beta}\right)^{2}}-\frac{p_{\alpha} z_{\alpha}^{2}-p_{\beta} z_{\beta}^{2}}{p_{\alpha}-p_{\beta}}},
$$

in terms of $4 c$ independent variables $\left\{\boldsymbol{q}_{\alpha}, z_{\alpha}, p_{\alpha}\right\}$. These variables preserve the geometric constraints defined by Eqs. (2) and (4) and provide an explicit, local expression for the tensions via the YL law and Eq. (7). [Note that these expressions assume $p_{\alpha} \geq 0$ : since YL law involves only pressure differences, Eq. (3) defines pressures only up to an additive constant, which can be used to ensure that all $p_{\alpha}$ 's are non-negative.] Our parametrization shows that centroids of edge circular arcs that meet at a vertex are collinear at mechanical equilibrium, as previously noted in foams [42]. We note that Eqs. (6) and (7) are explicitly invariant with respect to rescaling of $p_{\alpha}$.

The dual variables defined above can be interpreted geometrically in terms of the generalized Voronoi construction that can be used to generate equilibrium CAP tilings [see Fig. 2(b)]. Specifically, we define the distance from point $\boldsymbol{r}$ in the plane to a generating point $\alpha$ positioned at $\left\{\boldsymbol{q}_{\alpha}, z_{\alpha}\right\}$, with $z_{\alpha}$ denoting the height above the $x y$ plane, as shown in Fig. 2(b), as

$$
d_{\alpha}^{2}(\boldsymbol{r})=\left|\boldsymbol{r}-\boldsymbol{q}_{\alpha}\right|^{2}+z_{\alpha}^{2} .
$$

Each edge $\alpha \beta$ of an equilibrium CAP tiling is the locus of points $\boldsymbol{r}$ that satisfy $p_{\alpha} d_{\alpha}^{2}(\boldsymbol{r})=p_{\beta} d_{\beta}^{2}(\boldsymbol{r})$ relative to the corresponding pair of generating points. The general solution for the edge geometry is the circle with centroid $\rho_{\alpha \beta}$ and radius $R_{\alpha \beta}$ given by Eqs. (6) and (7), respectively. The connection with the standard Voronoi construction becomes evident when all $p_{\alpha}$ are set to one and all $z_{\alpha}$ are set to zero, in which case the edge becomes a straight line bisecting $\boldsymbol{q}_{\alpha}-\boldsymbol{q}_{\beta}$. In that case, $\boldsymbol{q}_{\alpha}$ are simply the vertices of the dual Delaunay triangulation. (In the constant pressure limit, $z_{\alpha}^{2}$ describe the isogonal deformations of the polygonal cell arrays corresponding to equilibrium tension networks [18].)

We note that one can alternatively use the sum of $\left[p_{\alpha} d_{\alpha}^{2}(\boldsymbol{r})-p_{\beta} d_{\beta}^{2}(\boldsymbol{r})\right]^{2}$ on all edges (see Appendix B) as an objective function analogous to Eq. (5) to be minimized with respect to the dual variables $\left\{\boldsymbol{q}_{\alpha}, z_{\alpha}, p_{\alpha}\right\}$. The two minimization approaches are equivalent: in Appendix B we show algebraically that $\left(\left|\boldsymbol{r}_{\alpha \beta}(n)-\boldsymbol{\rho}_{\alpha \beta}\right|-R_{\alpha \beta}\right)^{2}=$ $A_{\alpha \beta}\left[p_{\alpha} d_{\alpha}^{2}(\boldsymbol{r})-p_{\beta} d_{\beta}^{2}(\boldsymbol{r})\right]^{2}$ (with a prefactor $A_{\alpha \beta}$ computed in Appendix B). We note that the use of dual variables Eqs. (6) and (7) to express $\boldsymbol{\rho}_{\alpha \beta}$ and $R_{\alpha \beta}$ ensures that even when the latter two diverge as they do for a straight edge, the corresponding terms in Eq. (5) stay finite and the minimization is well defined.

The reduced variable representation given by Eqs. (6) and (7) makes the task of finding the best-fitting equilibrium CAP tiling relatively straightforward. Given a gray scale image of cellular boundaries in an epithelial monolayer, corresponding to a large number of cells $(\sim 20-1000)$ with interfaces resolved at a pixel level, we proceed by minimization of Eq. (5) with respect to reduced variables. Minimization of Eq. (5) is a nonlinear optimization problem subject to linear inequalities on $z_{\alpha}^{2}$,s that ensure positivity of the argument of the square root in Eq. (7). Furthermore, we constrain all pressure values to be absolute-i.e., $p_{\alpha} \geq 0$ - to ensure positive distance values in the generalized Voronoi construction. We solve the problem computationally using MATLAB's implementation of the interior-point algorithm. The simple choice of starting the iteration with an estimate of $\left\{p_{\alpha}, \boldsymbol{q}_{\alpha}, z_{\alpha}\right\}$ obtained from the observed set of network angles (as explained in detail in Appendix C) was found to produce reliable convergence. For approximately 1000 simulated cells, the minimization takes $\sim 5 \mathrm{~min}$ on a MacBook Air laptop.

\section{VALIDATION OF THE VARIATIONAL INFERENCE METHOD}

To evaluate the performance of the VMSI method, we tested it against synthetically generated data. Specifically, we performed a systematic sensitivity analysis of our algorithm, and two previous inference schemes [32,35], for cellular networks in the presence of variable noise and pressure differentials. Under low noise and small intercellular pressure differentials, all algorithms performed equally well. However, as exemplified by Fig. 3, we found the variational inference to be significantly more accurate in the face of moderate to high measurement noise and large pressure differentials. (See Appendix D for more details on the performance of the VMSI algorithm in comparison to Refs. [32,35].) 

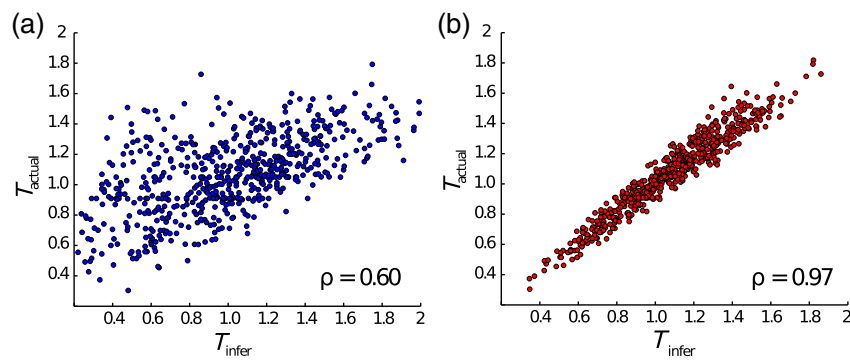

FIG. 3. Validation of VMSI for force inference using synthetic data. (a) Comparison of actual and inferred tensions based on the matrix-inverse-type inference method introduced in Ref. [29] made overdetermined using measured edge curvature data, as in Ref. [35]. $10 \%$ noise was added to the synthetic input data (see Appendix D for details). $\rho$ denotes Pearson's correlation coefficient. (b) Same as (a) but using the VMSI on the same synthetic data. Note the significant increase of the correlation coefficient.

The key to the improved performance is the overconstrained nature of the present formulation of the mechanical inference problem, which combines the estimation of geometric parameters with force inference into a single variational analysis of imaging data. We found empirically that the redundancy of parametrization not only stabilizes the inference in the presence of noise, it also allows us to infer forces at the boundary using just the cell morphology in the 2D bulk. In short, the method is local and thus immediately applicable to inference of tissue-scale stress, as we discuss below.

\section{LOCAL AND GLOBAL STRESS INFERENCE AND ITS in vivo CORRELATES}

The inferred set of interfacial tensions and cellular pressures allows us to construct a stress tensor for an epithelial layer in mechanical equilibrium. Over the 2D "bulk" of cell $\alpha$, the stress is isotropic and constant: $\boldsymbol{\sigma}=$ $p_{\alpha} \boldsymbol{\delta}$ ( $\boldsymbol{\delta}$ denotes the identity matrix) and $\boldsymbol{\sigma}=T_{\alpha \beta} \hat{\boldsymbol{r}}_{\alpha \beta} \otimes \hat{\boldsymbol{r}}_{\alpha \beta}$ on edge $\alpha \beta$. Averaging over the cell area $A_{\alpha}$,

$$
\overline{\boldsymbol{\sigma}}_{\alpha}=-p_{\alpha} \boldsymbol{\delta}+\sum_{\{\beta\}_{\alpha}} \frac{T_{\alpha \beta}}{2 A_{\alpha}} \int_{\boldsymbol{r}_{\alpha \beta}} d r \hat{\boldsymbol{r}}_{\alpha \beta} \otimes \hat{\boldsymbol{r}}_{\alpha \beta},
$$

where $\{\beta\}_{\alpha}$ denotes all cells connected to cell $\alpha$ and integration is taken along the circular arcs at the perimeter of cell $\alpha$ (see Ref. [43]). In practice, it is useful to coarse grain the tensor by averaging over neighboring cells to obtain the continuum stress tensor [44]. In the following section, we verify the utility of the proposed mechanical inference by comparing the inferred stress tensor to known biological correlates of stress.

We now assay the utility of the VMSI algorithm to imaging data from in vivo epithelial monolayers. Ideally we would test VMSI against direct measurements of stress. Presently, the most reliable readout of local stress in live tissue is provided by observed levels of (fluorescently labeled) junctional myosin, which has been previously demonstrated to correlate with local interfacial tension measured by laser ablation [2]. We shall carry out the comparison between inferred stress and observed myosin level first on the scale of cells, then on the scale of the whole tissue, using the data on early Drosophila embryonic development.

During the initial stages of Drosophila embryonic development, the ellipsoidal monolayer of epithelial cells forming the embryo undergoes a series of nontrivial mechanical transformations. Immediately following the formation of the ventral furrow-the first step of gastrulation-the Drosophila embryo undergoes germ-band extension (GBE): a major morphogenetic movement involving a convergent extension of the lateral ectoderm, which approximately doubles its length along the embryo's anterior-posterior (AP) axis. This process has been demonstrated to be driven by the activity of the junctional pool of myosin II, which exhibits a nonuniform and anisotropic distribution on the surface of the embryo, in particular, forming contractile supercellular cables that run along the dorsal-ventral (DV) axis of the embryo [36,38,45]. Laser ablation assays have demonstrated that these myosin cables, associated with DV oriented edges, exhibit significantly higher cortical tension than AP oriented cell junctions $[45,46]$. The quantitative relation between myosin and mechanical stress was further elaborated in our earlier study of morphogenetic flow [36], which demonstrated that a symmetric 2D tensor $m_{a b}$ describing coarse-grained distribution of myosin is a useful proxy for the stress tensor.

Applying VMSI to the embryonic epithelium images [36], we found that cell-array geometry observed over the first 60 min of convergent extension is quite well approximated by an equilibrium cell network, $\langle E\rangle \approx 1$, which means our best-fit equilibrium CAP geometry differs from the image segmentation by on average one pixel per edge (with the median pixel count per edge being 25). The complete distribution of residuals over time can be found in Appendix F.

Figure 4 shows results of the analysis on the lateral ectoderm during GBE. Qualitatively, the inferred stress exhibits anisotropic stress cables that run along the DV axis in agreement with previous studies [45]. For a quantitative comparison, we compute the correlation coefficient of tension inferred on individual cellular interfaces with the myosin line density measured on the same interfaces: a histogram of the calculated results for each time point is shown in Fig. 4(c). The mean correlation coefficient, $\rho \sim 0.4$, is a twofold improvement over the earlier "matrix inverse" method [29].

The observed correlation indicates that the inference method is picking up underlying mechanical effects, albeit statistically. There are, however, numerous sources of noise that weaken correlation. To name two, (i) while the analysis 


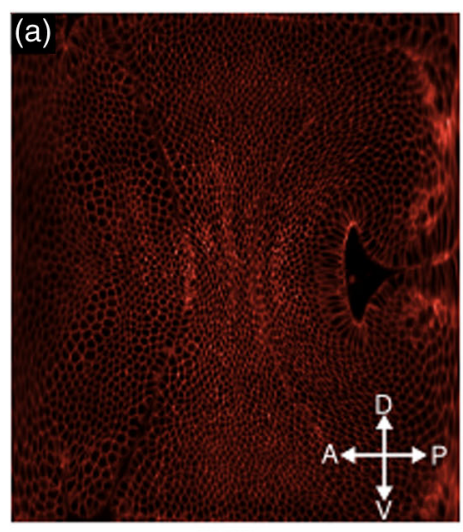

(d)

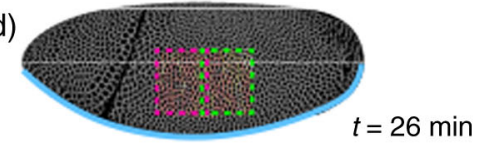

(10)
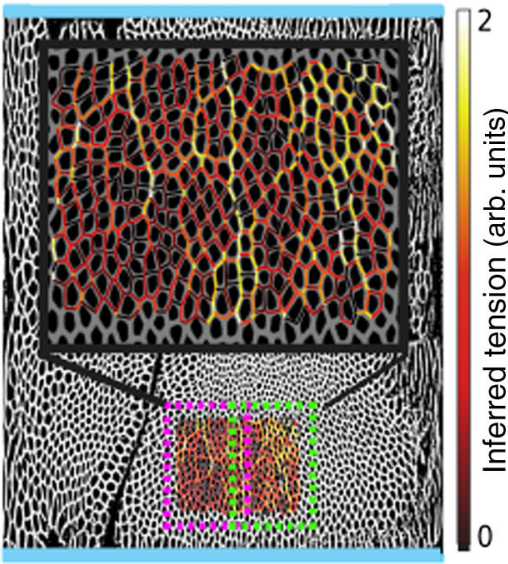

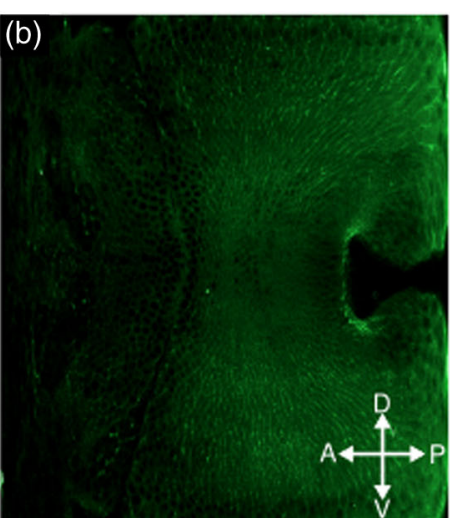

(e)
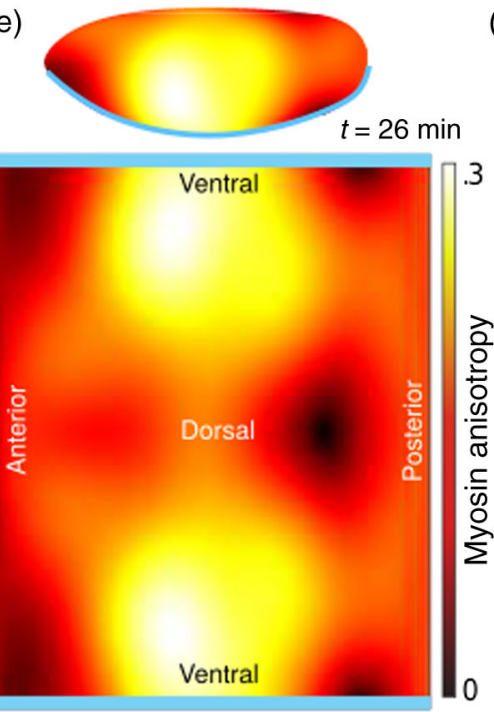

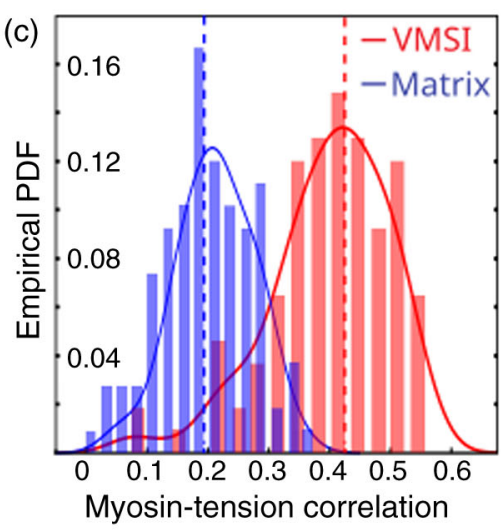

(f)

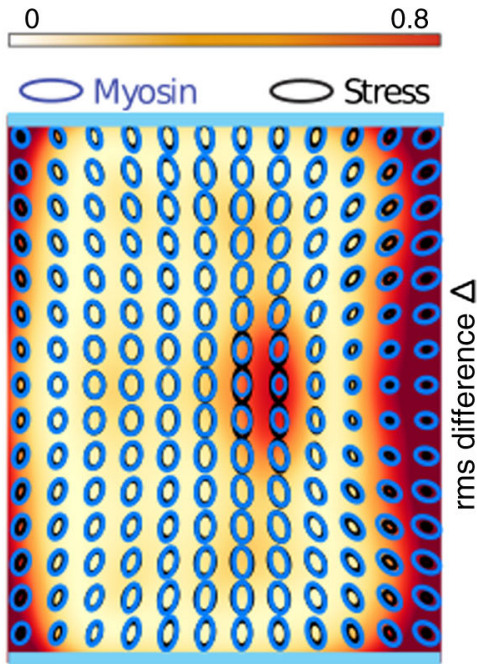

FIG. 4. Stress inference results compared to measured myosin distribution for the germ-band extension in Drosophila embryo. (a),(b) Raw in toto images showing fluorescent-labeled membrane [mCherry, shown in (a)] and myosin [Sqh-GFP, shown in (b)] obtained using light sheet microscopy and unrolled into the plane using Imsane [47]. Time corresponds to roughly 26 min after cephalic furrow (CF) formation. (c) The correlation between myosin and inferred tensions comparing the matrix-inverse-based method defined in Ref. [29] and the VMSI algorithm during the first 40 min of GBE. (d) An example of force inference, combining two overlapping image frames used for the analysis, shown directly on the embryo and on a cylindrical projection with the ventral line cut and mapped onto the top and bottom edges of the image with the dorsal side along the midline. Inset: Color-coded relative edge tension (in arbitrary units, arb. units) inferred by the VMSI algorithm. The inferred mechanics displays stress cables as expected for the lateral region of the embryo at the time considered. (e) The mesoscopic anisotropy of myosin, 26 min post CF formation, shown on the embryo and its cylindrical projection. Anisotropy is largest in the lateral region. The principal axis of myosin in this region points along the DV axis. (f) The spatial distribution of the normalized rms difference $[\Delta(r)]$ between the VMSI inferred stress tensor and the measured total myosin tensor (at 26 min post CF). Both tensor fields are represented as ellipses for direct comparison. The large discrepancy $(\Delta \sim 0.8)$ found in the vicinity of the anterior and posterior poles (mapped to the left and right edges of the cylindrical projection) is due to the poor imaging of the poles. The large discrepancy in the center of the dorsal region is real and can be explained by the difference between the total and "balanced" myosin distributions as explained in the text.

was carried out on a single snapshot, cell geometry is fluctuating on the timescale of seconds, and (ii) linear density of (fluorescent-labeled) myosin is not an exact measurement of line tension. Most importantly, the assumption that cells are in a mechanical equilibrium is at best only an approximation: in the case of GBE, there is a mean morphogenetic flow of cells indicating the presence of unbalanced local forces within the tissue [36]. Below, we demonstrate that accounting for this systematic deviation from the mechanical equilibrium effect improves the correlation between inferred stress and measured myosin distribution at the mesoscopic scale.

Edge-by-edge comparison is the most exacting test, as it is sensitive to local fluctuations. Myosin distribution, however, also exhibits nontrivial variation over the surface of the embryo [see Fig. 4(e)] and it is informative to compare it with the inferred stress tensor coarse grained via Eq. (9). In constructing the latter, it is helpful that VMSI inference can be carried out locally on partially overlapping image frames and "stitched" [depicted in Fig. 4(d); see 
Appendix E for details] into a continuous coarse-grained stress field for the whole surface of the embryo. In Fig. 4(f) we compare the inferred coarse-grained stress tensor with the measured coarse-grained myosin tensor [36]. Both myosin and inferred stress are enriched in the lateral ectoderm and are anisotropic along the DV axis, with the quantitative agreement at the level of about $60 \%$ [which corresponds to average $\Delta \sim 0.4$ in Fig. 4(f)].

To more exactly quantify the difference between the measured myosin tensor $\boldsymbol{m}$ (as defined in Ref. [36]) and the inferred stress tensor $\boldsymbol{\sigma}$, we defined a normalized rootmean-square (rms) deviation:

$$
\Delta(r) \equiv\left[\frac{\left\langle\operatorname{Tr}(\boldsymbol{m}-\lambda \boldsymbol{\sigma})^{2}\right\rangle_{r}}{\left\langle\operatorname{Tr}\left(\boldsymbol{m}^{2}\right)\right\rangle_{r}}\right]^{1 / 2},
$$

where $\langle\cdots\rangle_{r}$ denotes averaging over a coarse-grained region centered at location $r$ and $\lambda$ is the unknown overall scaling factor relating myosin and stress, which we chose so as to minimize the global average of $\Delta$. Hence, $1-\Delta(r)$ is the measure of local agreement (in both magnitude and anisotropy) between of $\boldsymbol{\sigma}$ and $\boldsymbol{m}$ within a coarse-graining region centered at position $r$.

\section{A. Comparison of inferred stress to balanced myosin tensor}

In general, the inferred stress tensor is consistent with the measured myosin tensor-both exhibit strong anisotropy localized to the lateral ectoderm, with principal axis along the DV axis. The most substantial disagreement between the inferred stress and measured myosin tensors, as shown in Fig. 4(f), is localized at the center of the dorsal side of the embryo; myosin is spatially inhomogeneous along the DV axis (high at the lateral sides) in contrast to the inferred stress tensor, observed to be constant along the DV axis. A plausible explanation for this discrepancy is that the stress tensor is inferred under the assumption of mechanical equilibrium, while the measured myosin tensor is not fully balanced as it is known to drive the early morphogenetic flow [36].

Our previous study [36] related observed mesoscale myosin distribution $\boldsymbol{m}$ and observed morphogenetic flow, by focusing on the divergence of the myosin tensor $\nabla \cdot \boldsymbol{m}$ which corresponds to the "unbalanced" internal stress within the tissue that generates cellular flow. (Note that, following Ref. [36], we assume $\boldsymbol{\sigma} \sim \boldsymbol{m}$.) However, only a fraction of myosin contributes the unbalanced stress, the rest generates internal stress which obeys force balance: $\boldsymbol{m}=\boldsymbol{m}_{U}+\boldsymbol{m}_{B}$. The "balanced" fraction of myosin $\boldsymbol{m}_{B}$ is, by definition, divergenceless, $\nabla \cdot \boldsymbol{m}_{B}=0$, and is explicitly the component of the overall myosin tensor that is expected to correlate with the predictions of VMSI-based inference.

To decompose measured myosin tensor [36] into "balanced" and "unbalanced" components, we note that any 2D symmetric tensor can be represented by a vector field $\vec{u}$ and a scalar field $\phi$ in the following form:

$$
m^{a b} \equiv\left[\nabla^{a} u^{b}+\nabla^{b} u^{a}\right]+\varepsilon^{a c} \varepsilon^{b d} \nabla^{c} \nabla^{d} \varphi,
$$

where we have introduced component notation for convenience-i.e., $a, b$ index spatial component. $\varepsilon^{a c}=$ $-\varepsilon^{c a}$ denotes the antisymmetric unit tensor. Conversely, the divergence of the last term is zero and it can be identified as $m_{B}^{a b}$, the balanced component of the myosin tensor. Taking the divergence of Eq. (11) yields a partial differential equation for the vector field $u^{b}$ :

$$
\nabla^{2} u^{b}+\nabla^{b} \nabla^{a} u^{a}=\nabla^{a} m^{a b},
$$

which was solved using the same method as in Ref. [36]; see Sec. VII. [Strictly speaking, Eq. (12) defined $u^{a}$ only up to harmonic gradients, $u^{a} \rightarrow u^{a}+\nabla^{a} \psi+\varepsilon^{a c} \nabla^{c} \omega$, with $\nabla^{2} \psi=\nabla^{2} \omega=0$. Since the only solution to the latter equations on a closed surface of genus zero is a constant, our solution for $u_{a}$ is unique.] Equations (11) and (12) provide an explicit determination of the balanced component of myosin tensor:

$$
m_{B}^{a b} \equiv m^{a b}-\left[\nabla^{a} u^{b}+\nabla^{b} u^{a}\right] .
$$

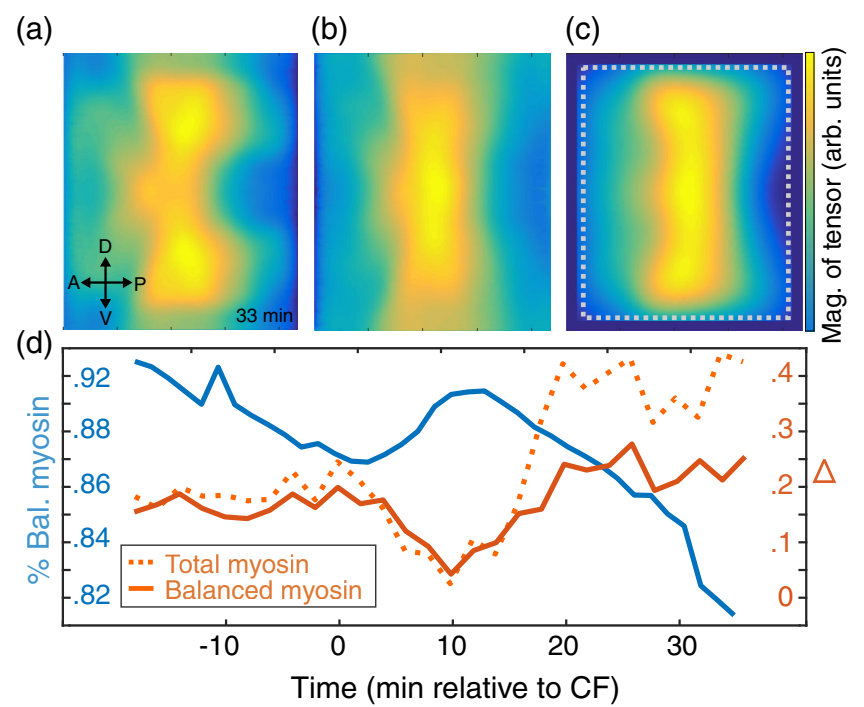

FIG. 5. Balanced myosin versus total myosin. (a) The magnitude of the myosin tensor measured in Ref. [36] $33 \mathrm{~min}$ after the formation of the ventral furrow. (b) Same for the balanced component myosin tensor, defined by Eq. (13), at the same time. Note that the balanced myosin is approximately constant along the DV axis in contrast with the total myosin displayed in (a). (c) The magnitude of the inferred stress tensor at the same time point. Note greater similarity with (b) than with (a). The dotted gray box shows the region that was amenable to image segmentation. (d) The average misalignment Eq. (10) between inferred stress and myosin tensors [balanced (total) displayed as solid (dashed) orange lines]. The balanced fraction of myosin is shown in blue. As expected, the inferred stress tensor provides a better approximation for the balanced component of myosin tensor then for the total. 
Figure 5 displays the distribution of $m_{B}^{a b}$ on the surface of the embryo and compares it with the total myosin distribution and the inferred stress. As shown in Fig. 5(d), balanced myosin dominates, accounting for more than $80 \%$ of the total, but the unbalanced component increases with time, especially upon the onset of the GBE [10 min post Cephalic Furrow (CF)]. Specifically, during GBE the alignment of our inferred stress tensor relative to the total myosin tensor decreases to $\sim 60 \%$, consistent with the higher fraction of unbalanced myosin and thus morphogenetic flow. Most interestingly, we find that removing the unbalanced component from the myosin distribution being compared to the inferred stress substantially increases the agreement between the two [see Fig. 5(d)]. This is expected since the inference algorithm assumes mechanical equilibrium and therefore can only pick up the balanced part of stress.

\section{B. Principal stress axis predicts mitotic orientation}

Let us now provide an example of how our variational stress inference can be used to study interesting questions concerning mechanical control of biological phenomena. It is known that the spatiotemporal patterning and orientation of cell divisions plays an important role in morphogenesis [48]. Mitotic domains of synchronously dividing cells partition the Drosophila embryo in a highly regular manner that directly shapes eventual larval segments [49]. Additionally, the patterning of mitotic spindle orientation has been suggested to contribute to elongation of the posterior region of the lateral ectoderm [50]. While the upstream signal that instructs the orientation of the cell cleavage plane is unknown, studies suggest that mechanical tension within the tissue contributes to spindle alignment [51-53] in the dividing cell. Additional evidence that interphase cell strain orients mitotic cells by recruitment of Mud (a protein important for spindle orientation) to tricellular junctions was reported in Ref. [54].

To test the hypothesis that the cell cleavage axis tends to align with local tension within cells, we analyzed 70 tracked divisions in mitotic domains 6 and 11 (as defined in Ref. [49]) during the late phase of GBE [20-35 min post cephalic furrow (CF) formation]. Figures 6(a) and 6(b) provide an example of using the VMSI method to infer tensions in individual interfaces of a cellular network. The cell cleavage axis was compared directly to the orientation of the tension axis determined from the inferred stress tensor, Eq. (9). We found that cell cleavage indeed correlates strongly with inferred tension and that the principal axis of stress is a much better predictor of spindle orientation at the time of division than the commonly used "long axis" defined directly by cell elongation. Furthermore, the VMSI-based inference was more accurate (based upon $95 \%$ confidence intervals using $t$-test) than the earlier "matrix inverse" approach of Ref. [29]. The improved accuracy is due to the VMSI's ability to capture large (a)

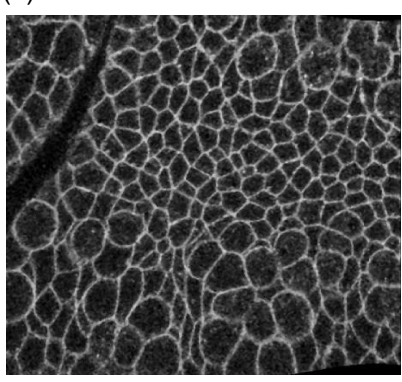

(c)

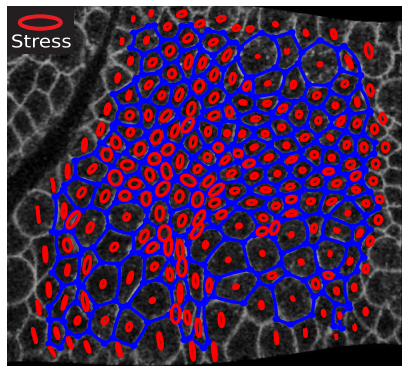

(b)

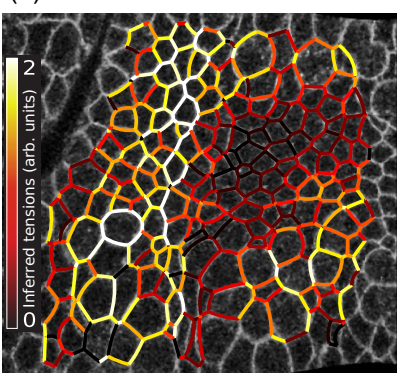

(d)

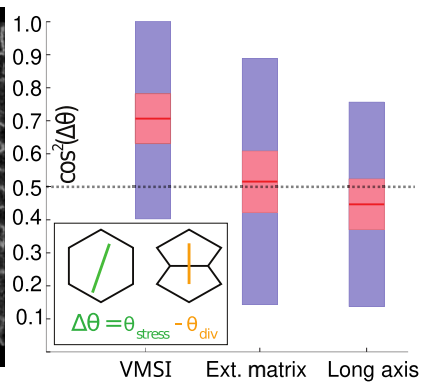

FIG. 6. Correlation of inferred stress and cell-division axis. (a) Confocal image of the Drosophila lateral ectoderm, near the cephalic furrow, during the late phase of germ-band extension (GBE) at the onset of cell divisions, approximately $45 \mathrm{~min}$ after the formation of the cephalic furrow. (b) An overlay of inferred tensions on the CAP array. Note that a low-tension edge (dark red) meets two high-tension edges; the latter meet at an angle close to $\pi$ while low-tension edge is nearly perpendicular. (c) Same as (b) but with the average stress tensor for each cell plotted as an ellipse. The major or minor axis of the ellipse corresponds to the principal axes of stress. (d) Comparison of the cell cleavage axis for cell division events (within mitotic domains 6 and 11 [49]) and the principal (extension) axis of inferred local stress (using different methods). Stress for mitotic cells was estimated $5 \mathrm{~min}$ before the registered time of division. While VMSI-based analysis finds significant correlation, predictions based on the extended "matrix inverse" $[29,35]$ or cell elongation axis are not distinguishable from random (shown as dashed gray line).

pressure differentials between cells in morphologically heterogeneous cell arrays, as exemplified by Fig. 6(a).

\section{DISCUSSION}

The VMSI algorithm described here was based on the model that assumed that (i) the 2D epithelial cell array is instantaneously in an approximate mechanical equilibrium and (ii) cell mechanics can be approximated by the balance of cytoskeletal tension localized at cell interfaces (and varying from one edge to another) and the effective areal pressure (preventing collapse of the apical surface of cells). Together, these assumptions place a nontrivial constraint on cell geometry that is readily testable solely on the basis of imaging data. The existence of local geometric constraints facilitated the formulation of a local mechanical inference 
scheme that combined the estimation of stress with the simultaneous determination of the best-fit cell geometry from imaging data. The assumption of mechanical equilibrium implies that the body force is zero locally within the tissue; hence our inference provides us a measurement of the local stress tensor, not the underlying forces. The method was observed to be a significant improvement over similar methods, both at the scale of individual cells and at the mesoscopic scale. We found much stronger agreement in between the coarse-grained mesoscopic stress and myosin relative to the comparison at the cellular scale.

While our analysis focused on cellular arrays with threefold vertices, our arguments generalize to an admixture of higher-order vertices. A fourfold vertex corresponds to a removal of an edge from a threefold network resulting in the coalescence of a pair of threefold vertices: two force balance constraints on the array are lost when two vertices merge into one. (The loss of the curvature measurement on the removed edge is offset by the reduction in the number of edge tension "unknowns.") However, the effect is localized to the four adjacent cells while, critically, VMSI on any 4 cells is overconstrained by $3 \times 4=12$ equations. Hence, loss of two constraints will not destabilize the local (and thus global) inference. Mathematically, VMSI would remain overconstrained (although to a lesser degree) even if all of the vertices were fourfold. The utility of the geometric constraints identified in the present study is that they provide a direct way to test whether an observed cell array at least approximately satisfies the constraints of mechanical equilibrium, purely on the basis of cell morphology and image analysis.

In addition to testing the validity of the VMSI-based force inference, our analysis of myosin and inferred stress distributions in Drosophila embryo has revealed that despite the dynamical nature of GBE, the epithelial shell of the embryo maintains approximate mechanical equilibrium, in the sense that mechanical stress associated with the observed myosin distribution is mostly (at the $80 \%$ level) balanced internally and does not contribute to cellular flow. This result also holds at the cellular scale, albeit with less statistical significance. The above conclusion is reached by a direct analysis of the measured myosin tensor, Eq. (13). Quite remarkably, the presence of this balanced internal stress is also correctly inferred from the VMSI-based analysis of cell geometry across the surface of the embryo. The conclusion that tissue flow coexists with approximate internal force balance within a rearranging array of cells provides an interesting insight into the mechanics of tissues.

According to Ref. [36], the unbalanced myosin acts as a driver of global morphogenetic flow. Our analysis above decomposed myosin into balanced and unbalanced components; regions with substantial unbalanced to balanced fractions are then putative "drivers" of the overall morphogenetic flow. Disregarding the errors at the anterior and posterior poles that arise from image resolution issues, the largest deviation from mechanical balance is found along the dorsal surface [see Figs. 5(a)-5(c) and Fig. 4(f)], where total myosin falls below the level needed for internal force balance, as predicted by the balanced component. This suggests an important role for DV patterning in the convergent extension flow. We believe the ability to estimate global patterns of balanced and unbalanced stress on arbitrary two-dimensional surfaces opens up a novel method in which one can identify the factors that drive morphological change.

We expect the VMSI-based force inference to be immediately useful for experimentalists studying tissue mechanics and the mechanics of morphogenesis of entire organs.

\section{MATERIALS AND METHODS}

Confocal microscopy.-Raw data shown in Fig. 4 were taken on a Leica SP8 confocal microscope equipped with two HyD detectors, a $40 \times$, NA 1.1 water immersion objective, and $561 \mathrm{~nm}$ laser line.

Light sheet imaging.-In toto images for Figs. 5 and 6 were taken on a custom-built multiview light sheet microscope described in Ref. [55]. Briefly, the setup consisted of two excitation and two detection arms. On each detection arm, the microscope was equipped with a water-dipping lens (Apo LWD 25×, NA 1.1, Nikon Instruments, Inc.), a filter wheel (HS-1032, Finger Lakes Instrumentation LLC) with emission filters (BLP02-561R-25, and BLP01-488R-25, Semrock, Inc), a tube lens (200 mm, Nikon Instruments, Inc.), and a sCmos camera (Hamamatsu Flash $4.0 \mathrm{v} 3$ ). In this way an effective pixel size of $0.26 \mu \mathrm{m}$ was achieved. Illumination consisted of a water-dipping objective (CPI Plan Fluor 10×, NA 0.3, Nikon Instruments, Inc), a tube lens (same as above), a scan lens (S4LFT0061/ 065, Sill optics $\mathrm{GmbH}$ Co. KG), and a galvanometric scanner (6215h Cambridge Technology, Inc.). Illumination was based on laser lines (06-MLD $488 \mathrm{~nm}$, Cobolt AB, and 561 LS OBIS $561 \mathrm{~nm}$, Coherent, Inc.). 3D volumes were generated by translating samples through the sheet using a linear piezostage (Physik Instrumente P-629.1CD controlled by C-867). Multiple views were generated by a rotation stage (Physik Instrumente U-628.03, C-867 controller), combined with a linear actuator (M-231.17, C-863 controller). Electronic control of the microscope was based on $\mu$ MANAGER [56] and a custom-written MATLAB code. Fusion of individual views taken at $45^{\circ}$ angles was carried out using FIJI multiview fusion plug-ins [57]. Cartographic projections where generated using ImSaNe [47], a custom MATLAB package for analyzing 3D images [44].

Fly stocks.-Sqh-GFP; membrane-mCherry.

Numerical solution of Eq. (12).-A 2D triangulated mesh of the embryo surface was constructed using ImSaNe [47], and FELICITY [58] — a finite element software package for MATLAB-was utilized to solve partial 
differential equations and compute surface derivatives. See Ref. [36] for a detailed description of the method.

Image segmentation.-In vivo data were segmented using a custom pipeline implemented in MATLAB, available on Github [39] under the "seg" workspace. ilastik, a supervised machine-learning classifier, was used as a preprocessing step for each image, followed by the application of a Laplacian of Gaussian filter (MATLAB), with the kernel size chosen to be $\sim 5$ cell diameters. The resultant image was segmented using the watershed algorithm (MATLAB).

Vertices were defined as branch points of the resultant skeletonization-edges are segmented as the set of boundary pixels that run between two such branch points. Our CAP is parametrized by not only vertex position but also edge curvature. Each edge was fit to a circular arc using the Pratt method, which is robust for small angle samplings of the underlying circle. Interfacial myosin concentration was measured by dilation of each segmented edge by 2 pixels and averaging over the resulting set of pixels in the myosin channel. All segmentation information is stored within a custom data structure and can be immediately used for the VMSI inference. The code needed for the VMSI inference can be found at the same aforementioned Github repository [39] under the "fitDual" workspace.

Cell divisions during late germ-band extension were registered by tracking cells. Cell tracking was achieved by computing pixel overlaps between segmented cells in subsequent time points-cells were paired based upon the cell they most overlap with in the succeeding frame. Mitotic cells were defined as tracking events where two cells overlapped with one in the previous time point. The tracking was manually curated to ensure no false divisions were called. The long axis and the principal stress axis were computed at approximately $5 \mathrm{~min}$ before the tracked cell cleavage event. Supplemental figures pertaining to the dynamics of cellular geometry proceeding the division are included in Appendix F.

$3 D$ reconstruction.- ImSaNe [47] was used to measure, parametrize, and store the surface and embedding coordinates of the Drosophila embryonic surface. Segmentation of cells was done using the cylindrical mapping of the embryo. The 3D vertex positions were subsequently estimated using the embedding grids obtained from the ImSaNe algorithm.

We computed the mesoscopic myosin distribution in the same way as detailed in Ref. [36]. The output from the automated segmentation of myosin is a summation over microscopic nematic tensors of the form

$$
m^{a b}(\boldsymbol{r})=\sum_{\langle i, j\rangle} m_{i j} \hat{n}_{i j}^{a} \hat{n}_{i j}^{b} \delta^{2}\left(\boldsymbol{r}-\boldsymbol{r}_{i j}\right)
$$

This was averaged using a Gaussian filter.

\section{ACKNOWLEDGMENTS}

The authors gratefully acknowledge stimulating discussions with Madhav Mani, Idse Heemskerk, and Eric Wieschaus. The authors thank Yohanns Bellaiche and Boris Guirao for providing the code used in [32]. This work was supported by the NSF PHY-1220616 (B. I. S.) and NICHD 5K99HD088708-02 (S. J. S.).

\section{APPENDIX A: NONPLANARITY OF TENSION TRIANGULATIONS}

As stated in the main text, the tension triangulation "dual" to a CAP tiling is not planar. It follows from the definition of the dual triangulation-i.e., by rotating tension vectors which meet at a vertex by $\pi / 2$ - that the angles of each triangle are complements of angles at CAP vertices: $\bar{\phi}_{i \alpha}=\pi-\phi_{i \alpha}$. We note that if the edges of the polygonal tiling were straight, then $\sum_{i} \bar{\phi}_{i \alpha}=2 \pi$, corresponding to a planar triangulation as discussed in Ref. [18]. However, in an equilibrium CAP network, tension vectors acting at either end of an interface are no longer parallel: because of interfacial curvature, the tension vector acting on vertex $i$ (on edge $\alpha \beta$ ) is rotated by an angle $\Delta \varphi_{\alpha \beta}=$ $\ell_{\alpha \beta} / R_{\alpha \beta}$ relative to the tension vector at vertex $j$, where $\ell_{\alpha \beta}$ is the physical arc length of the circular edge. This results in the nonplanarity of the tension triangulation manifested by the "deficit" angle for each cell $\Delta \varphi_{\alpha}$ defined as the sum of curvature contributions from all edges that compose the cell:

$$
\Delta \varphi_{\alpha}=\sum_{\{\beta\}_{\alpha}} \frac{\ell_{\alpha \beta}}{R_{\alpha \beta}} .
$$

The deficit angle $\Delta \varphi_{\alpha}$ associated with cell $\alpha$ is the discrete Gauss curvature for the corresponding vertex in the dual triangulation. The dual tension triangulation is in one-toone correspondence with angles of the cellular array.

\section{APPENDIX B: REDUCED VARIABLES SOLVE GEOMETRIC CONSTRAINTS OF EQUILIBRIUM CAP ARRAYS}

In this Appendix, we show that reduced variables $\left\{\boldsymbol{q}_{\alpha}, z_{\alpha}, p_{\alpha}\right\}$ introduced in Eqs. (6) and (7) explicitly resolve geometric constraints implied by mechanical equilibrium, as observed for soap foams in Ref. [42]. We begin by noting that the tension vector $\boldsymbol{T}_{i, j}$ found in Eq. (1) is tangent to the circular arc radius, depicted in Fig. 1(c), and can be rewritten using the YL law in the form

$$
\boldsymbol{T}_{i, j}=\hat{z} \wedge\left[\boldsymbol{r}_{i}-\boldsymbol{\rho}_{\alpha \beta}\right]\left(p_{\alpha}-p_{\beta}\right),
$$

where the sign of $p_{\alpha}-p_{\beta}$ ensures the correct direction of the vector. Substituting into Eq. (1) we rewrite the force balance for vertex $i$ as 


$$
\left[p_{\beta}-p_{\alpha}\right] \boldsymbol{\rho}_{\beta \alpha}+\left[p_{\alpha}-p_{\gamma}\right] \boldsymbol{\rho}_{\alpha \gamma}+\left[p_{\gamma}-p_{\beta}\right] \boldsymbol{\rho}_{\gamma \beta}=0,
$$

which, quite remarkably, means that in mechanical equilibrium the centroids of all three interfacial circular arcs meeting at a vertex are constrained to be collinear.

Next we note that force balance equations rewritten in this form are trivially solved by $\boldsymbol{\rho}_{\beta \alpha}$ given by Eq. (6), as the left-hand side of the equation would have two factors of each $p_{\alpha} \boldsymbol{q}_{\alpha}$ with opposing signs.

In order for all three incident edges, corresponding to collinear centroids, to intersect at a single point uniquely defining a CAP vertex, their respective radii must obey

$$
\begin{gathered}
\Delta p_{\alpha \beta}\left(R_{\alpha \beta}^{2}-\boldsymbol{\rho}_{\alpha \beta}^{2}\right)+\Delta p_{\beta \gamma}\left(R_{\beta \gamma}^{2}-\boldsymbol{\rho}_{\beta \gamma}^{2}\right) \\
+\Delta p_{\gamma \alpha}\left(R_{\gamma \alpha}^{2}-\boldsymbol{\rho}_{\gamma \alpha}^{2}\right)=0,
\end{gathered}
$$

which imposes $v$ constraints on $e$ curvature variables. One can verify by direct substitution that $\boldsymbol{\rho}_{\beta \alpha}$ and $R_{\alpha \beta}$ defined by Eqs. (6) and (7) explicitly satisfy Eq. (B3). The appearance of $z_{\alpha}$ d.o.f. is explained by observing that, given any set of $R_{\alpha \beta}^{2}$ that solves Eq. (B3), we can generate another geometrically compatible cell array by transforming $R_{\alpha \beta}^{2} \rightarrow R_{\alpha \beta}^{2}+\left[\left(p_{\alpha} z_{\alpha}^{2}-p_{\beta} z_{\beta}^{2}\right) /\left(p_{\alpha}-p_{\beta}\right)\right]$. Hence, $z_{\alpha}$ provide an explicit parametrization of the $c$-dimensional manifold of solutions of Eq. (B3) that share the same set of edge centroids but have different radii.

We conclude this Appendix by directly relating the psuedoenergy Eq. (5) minimization, that we used to frame our variational approach, to the generalized Voronoi construction based on distances defined by Eq. (8). The latter construction corresponds to minimization of

$$
\begin{aligned}
\mathcal{E} & =\sum_{(\alpha, \beta)}\left(p_{\alpha} d_{\alpha}^{2}-p_{\beta} d_{\beta}^{2}\right)^{2} \\
& =\sum_{(\alpha, \beta)}\left(p_{\alpha}-p_{\beta}\right)^{2}\left[\left|\boldsymbol{r}-\boldsymbol{\rho}_{\alpha \beta}\right|^{2}-R_{\alpha \beta}^{2}\right]^{2} \\
& =\sum_{(\alpha, \beta)} A_{\alpha \beta}^{-1}\left[\left|\boldsymbol{r}-\boldsymbol{\rho}_{\alpha \beta}\right|-R_{\alpha \beta}\right]^{2},
\end{aligned}
$$

where all prefactors $A_{\alpha \beta}^{-1}=\left(p_{\alpha}-p_{\beta}\right)^{2}\left[\left|\mathbf{r}-\boldsymbol{\rho}_{\alpha \beta}\right|+R_{\alpha \beta}\right]^{2}$ which, upon reexpression in terms of dual variables using Eqs. (6) and (7) are seen to be explicitly positive, remain perfectly finite in the "straight edge" limit of $\left|p_{\alpha}-p_{\beta}\right| \rightarrow 0$. Hence, minimization of $\mathcal{E}$ is equivalent to minimization of $E$ in Eq. (5).

\section{APPENDIX C: INITIAL CONDITION FOR THE VMSI}

The initial condition for the variational parameters used in the minimization of Eq. (5) is obtained piecewise; we first approximate $\left\{\boldsymbol{q}_{\alpha}, p_{\alpha}\right\}$ independent of $z_{\alpha}$ by fitting edge centroids $\boldsymbol{\rho}_{\alpha \beta}$. As depicted in Fig. 2, the vector pointing from each arc's centroid to either of the attached vertices,

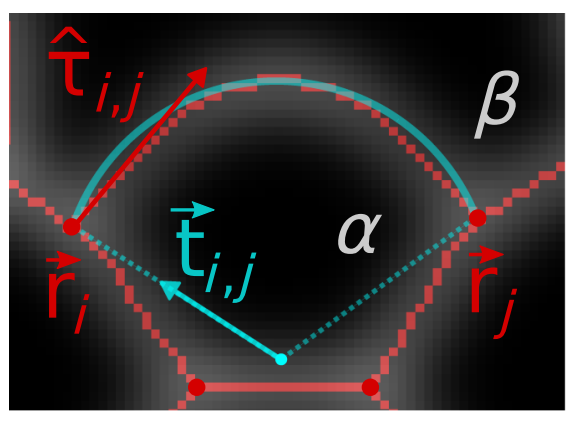

FIG. 7. An illustration of the definitions used in the procedure to find an initial estimate of $\left\{p_{\alpha}, \boldsymbol{q}_{\alpha}\right\}$, plotted on top of simulated data. The segmentation is shown in red while the idealized edge (to be fitted) is shown in light blue. The tangent vectors $\boldsymbol{\tau}_{i, j}$ are estimated from the segmentation. The minimization of $\mathcal{E}_{I}$ [Eq. (C1)] finds $\left\{p_{\alpha}, \boldsymbol{q}_{\alpha}\right\}$ minimizing the overlaps $\hat{\boldsymbol{t}}_{j, i} \cdot \hat{\boldsymbol{\tau}}_{j, i}$.

$\boldsymbol{r}_{i}-\boldsymbol{\rho}_{\alpha \beta}$, is orthogonal to the local tangent of edge $\boldsymbol{r}_{\alpha \beta}$. Analogous to the main text, we denote the measured tangent of edge $\alpha \beta$ at vertex $i$ as $\hat{\boldsymbol{\tau}}_{i, j}$.

The orthogonality between the fit radial vector $\boldsymbol{r}_{i}-\boldsymbol{\rho}_{\alpha \beta}$ and the measured $\hat{\boldsymbol{\tau}}_{i, j}$ can be imposed variationally on our parameters. It is convenient to introduce the shorthand notation $\boldsymbol{t}_{i, j} \equiv\left(p_{\alpha}-p_{\beta}\right) \boldsymbol{r}_{i}-\left[p_{\alpha} \boldsymbol{q}_{\alpha}-p_{\beta} \boldsymbol{q}_{\beta}\right]$; see Fig. 7 for a graphical depiction of the relevant definitions. Initial estimates for $\boldsymbol{q}_{\alpha}, p_{\alpha}$ are thus obtained by minimization of

$$
\mathcal{E}_{I}=\frac{1}{n_{e}} \sum_{\langle\alpha, \beta\rangle}\left[\boldsymbol{t}_{i, j} \cdot \hat{\boldsymbol{\tau}}_{i, j}\right]^{2}+\left[\boldsymbol{t}_{j, i} \cdot \hat{\boldsymbol{\tau}}_{j, i}\right]^{2} .
$$

The solution is constrained so that the ratio of the average magnitude of $\left|t_{i, j}\right|$ to the average pressure differential $\left|p_{\alpha}-p_{\beta}\right|$ equals the averaged measured radius of curvature in the image to ensure we do not pick the trivial solution $\left(\boldsymbol{q}_{\alpha}=\boldsymbol{q}_{\beta}, p_{\alpha}=p_{\beta}\right) . R_{\alpha \beta}$ is segmented from the image by fitting each edge to a circle using the Pratt method.

It is instructive to consider what happens on a straight edge, since the divergence of corresponding $R_{\alpha \beta}$ may seem problematic. In that case the two tangents at end points (see Fig. 7) are antialigned $\hat{\boldsymbol{\tau}}_{i, j}=-\hat{\boldsymbol{\tau}}_{j, i}$. Hence, minimization of the $\mathcal{E}_{I}$ contribution of that edge requires $\boldsymbol{t}_{i, j}|| \boldsymbol{t}_{j, i}$, which in turn requires that $\left|p_{\alpha}-p_{\beta}\right| \rightarrow 0$ so that $\boldsymbol{t}_{i, j} \cdot \hat{\boldsymbol{\tau}}_{i, j}=$ $\left(\boldsymbol{q}_{\beta}-\boldsymbol{q}_{\alpha}\right) \cdot \hat{\boldsymbol{\tau}}_{i, j}=0$. If all edges were straight this would result in a generalized Voronoi construction with $\boldsymbol{q}_{\alpha}$ as centroids (and $z_{\alpha}=0$ ). Critically, because our minimization variables $\left\{\boldsymbol{q}_{\alpha}, p_{\alpha}, z_{\alpha}\right\}$ remain finite and well defined in the limit of straight edges, there are no difficulties associated with divergent $R_{\alpha \beta}$.

The initial estimate of $z_{\alpha}^{2}$ is obtained from the variational parameters obtained above by inverting the linear set of equations defined by Eq. (7): 


$$
\begin{aligned}
p_{\beta} z_{\beta}^{2}-p_{\alpha} z_{\alpha}^{2}= & \frac{p_{\alpha} p_{\beta}\left|\boldsymbol{q}_{\alpha}-\boldsymbol{q}_{\beta}\right|^{2}-\left(p_{\alpha}-p_{\beta}\right)^{2} R_{\alpha \beta}^{2}}{p_{\alpha}-p_{\beta}} \\
= & {\left[p_{\alpha}-p_{\beta}\right]\left[\boldsymbol{r}_{i j}^{2}+\boldsymbol{q}_{\alpha} \cdot \boldsymbol{q}_{\beta}\right] } \\
& +2\left[p_{\beta} \boldsymbol{q}_{\beta}-p_{\alpha} \boldsymbol{q}_{\alpha}\right] \cdot\left[\boldsymbol{r}_{i j}-\frac{\boldsymbol{q}_{\alpha}+\boldsymbol{q}_{\beta}}{2}\right] .
\end{aligned}
$$

We have included (on the second line) the algebraic transformation explicitly eliminating $R_{\alpha \beta}$ from the system of equations determining $z_{\alpha}$.

Finally, we use the freedom of shifting all $p_{\alpha}$ by a constant, to ensure $p_{\alpha}>0$, and we fix the arbitrary overall scale of pressure and tension by imposing the constraint that $\sum_{\alpha} p_{\alpha} z_{\alpha}^{2}=1$.

\section{APPENDIX D: In silico BENCHMARK OF VMSI ALGORITHM}

To test the robustness of the VMSI inference, synthetic data were generated by initializing a triangular lattice of $\sim 120$ generating points $\boldsymbol{q}_{\alpha}$ within a rectangle of size $[1, \sqrt{3} / 2]$. Using generalized Voronoi construction defined in the main text, one can easily generate arbitrary CAP networks-i.e., for any set $\left\{\boldsymbol{q}_{\alpha}, p_{\alpha}, z_{\alpha}\right\}$-in mechanical equilibrium. Edges within the CAP network are defined by $p_{\alpha} d_{\alpha}^{2}=p_{\beta} d_{\beta}^{2}$ via Eq. (8). This procedure is repeated for each point $\boldsymbol{q}$. The minimum of $\left\{d_{\alpha}^{2}\right\}$ for each spatial location is taken-the net result is a scalar field that measures the minimum weighted distance away from any triangulation vertex, $d^{2}(\boldsymbol{r})=\min _{\alpha} d_{\alpha}^{2}(\boldsymbol{r})$. An example of the above procedure is shown in Fig. 8(a). Edges are "ridges" representing local maxima $d^{2}(\boldsymbol{r})$ that are found easily using the watershed algorithm-Fig. 8(b) shows an example. The resultant equilibrium network can then be
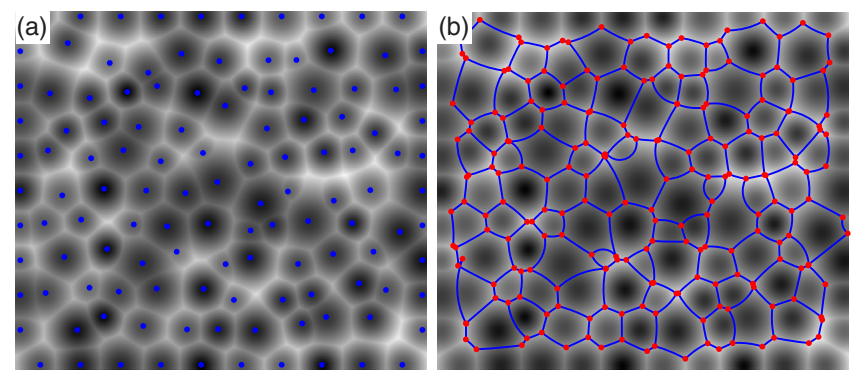

FIG. 8. (a) An example plot of the image of weighted distance from each generating point produced using MATLAB's bwdist function and the algorithm described. The locations of the generating points $\boldsymbol{q}_{\alpha}$ are shown as blue dots. (b) The resultant CAP network. The segmentation output from MATLAB's watershed algorithm is used to infer the triangulation topology. This is then passed into our forward equations to generate the exact positions of vertices and curvatures of edges. The result, using the original values for the generating points and the measured triangulation topology, is overlayed in blue over the original distance map. immediately calculated from the original parameters for $\left\{\boldsymbol{q}_{\alpha}\right\},\left\{p_{\alpha}\right\}$, and $\left\{z_{\alpha}\right\}$.

Intracellular pressures $p_{\alpha}$ were sampled from a uniform distribution to generate lattices of varying curvature. For simplicity, all $z_{\alpha}$ were held to zero to keep the number of simulation parameters small. White noise was added to both vertex position $\boldsymbol{r}_{i}$ as well as position of edge centroids $\boldsymbol{\rho}_{\alpha \beta}$ to simulate the effects of measurement and segmentation inaccuracy.

We benchmarked the relative efficacies, as a function of measurement noise and the contribution of pressure to mechanical balance, of three mechanical inverse algorithms: (i) the matrix inverse described in Ref. [32], (ii) an implementation of the extended matrix inverse on the curved cellular network as described in Ref. [35], and (iii) the proposed VMSI inference described in the main text.

The resultant correlation between the generated and inferred tensions and pressures is shown in Fig. 9 for the three algorithms, respectively. In general, we found all three algorithms performed well in the regime of low noise and low pressure - an example of a lattice in this regime is shown in Fig. 10(a). However, as pressure started to contribute non-negligibly to the mechanical balance of the network [see example in Fig. 10(b)], we observed that using only vertex positions (without curvature data) [32] produced noisier results, as shown in Fig. 9(a). By contrast, (our implementation of) the CellFIT algorithm [35] was more accurate in inferring mechanical parameters as pressure differentials increased, as inclusion of curvature data

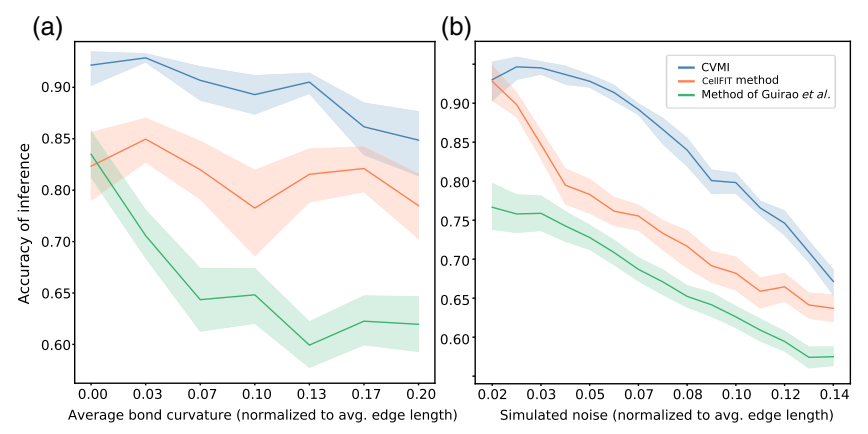

FIG. 9. Correlation between inferred tensions and pressures against the known synthetic values for three mechanical inference schemas-CVMI (blue), our implementation of the CellFIT method [35] (orange), and the inverse used within Ref. [32] (green) - as a function of the contribution of pressure to mechanical balance (a) and the strength of random perturbations to vertex position (b). Panel (a) represents a simulated lattice with constant noise of 0.03 (strength of perturbation is normalized to the average edge length). Similarly, panel (b) is taken at a finite average pressure differential of 0.03 (average ratio of edge length to radius of curvature). All algorithms were run on the same synthetic datasets. Each curve depicts the mean and variance of 100 networks of size $\sim 120$ cells generated with equivalent parameters as described in the text. 

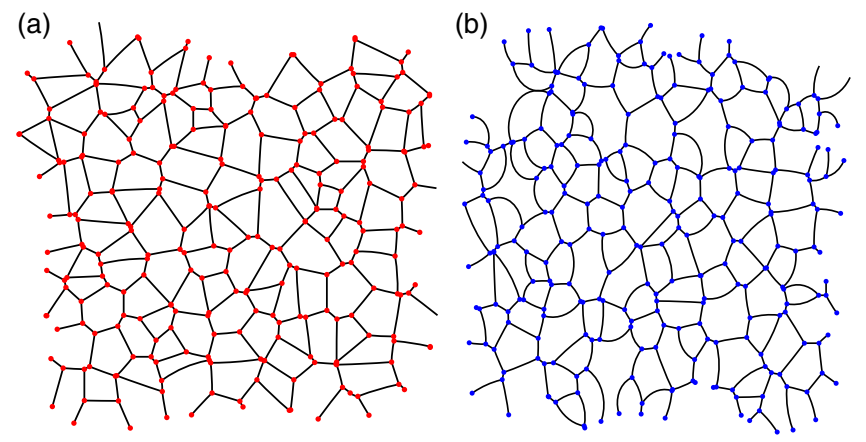

FIG. 10. Two examples of simulated CAP networks used in the in silico verification presented in Appendix D with varying average pressure differences. Networks (a) and (b) were generated with varying degrees of intracellular pressure differentials. The average edge curvatures, normalized to edge length-i.e., $R_{\alpha \beta} / r_{\alpha \beta}$-for (a) and (b) are 0.01 and 0.20 , respectively.

correctly imposes mechanical balance on the tangential edge network. However, we found the CellFIT algorithm more sensitive to simulated measurement noise than VMSI, seen in the decrease in accuracy given by the red curve in Fig. 9(b) as one moves to the higher simulated noise values. We conclude that the VMSI algorithm is more accurate in inferring the simulated mechanical parameters over the tested regime, albeit at the cost of the computation time required to perform the nonlinear least-squares regression.

\section{APPENDIX E: VMSI ALGORITHM APPLIED TO CURVED SURFACES}

The VMSI can be easily extended to formulate a tractable inference scheme for the balanced mechanical stress within a curved tissue's tangent plane. Because of the inclusion of edge curvature information, the inverse is extensively overdetermined, which allows one to simultaneously infer both bulk and boundary stress using information of just bulk geometry. Consequently, the global mechanical state can be "stitched" together by inferring stress on local patches of cells that can, with good approximation, be treated as planar.

The blue cell array depicted in Fig. 11(b) denotes the "true" apical surface of the epithelial tissue. Provided the area of interest is much smaller than the surface's radius of curvature, we can fit a well-defined tangent plane to the patch. Let $\boldsymbol{R}_{i}^{n}$ denote the 3D position of the $i$ th vertex within patch $n$-it is a matrix of size $3 v_{n}$, where $v_{n}$ is the number of vertices contained in patch $n$. The best-fit triad of vectors is obtained easily via an singular value decomposition, $\boldsymbol{R}_{i}^{n}=U_{i} \Sigma V_{n}^{T}$

The approximate planar graph of patch $n$, shown as the black cell array in Fig. 11(b), is obtained by projecting $\boldsymbol{R}_{i}^{n}$ onto the two principal components; VMSI is applied on the distortion. Importantly, the set of inferred tensions and pressures within each patch is unique up to an overall scale and thus there exists an undetermined relative scale

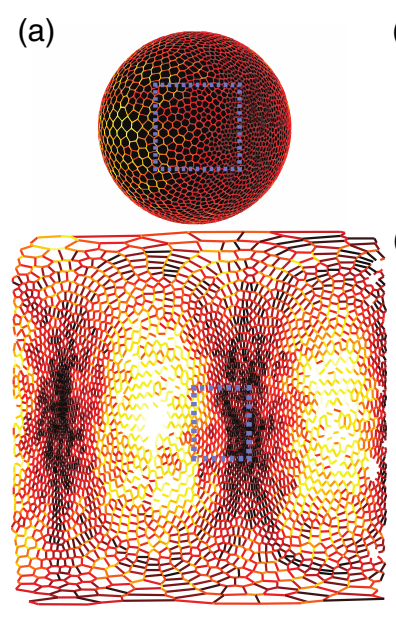

(b)

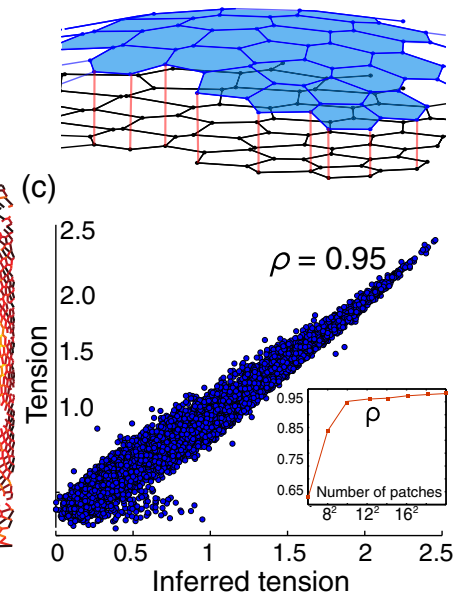

FIG. 11. (a) A synthetic spherical embryo of $~ 3000$ cells, plotted both in the embedded space, as well as the cylindrical unwrapping analogous to the embryonic data shown in the main text. Simulated interfacial tensions are plotted as a heat map in both domains. (b) Graphical depiction of the process used to infer stresses in the tangent plane of a small region of cells. All cells are segmented in 3D using Imsane [47]. Each local cellular patch is projected from the $3 \mathrm{D}$ embedding space onto the $2 \mathrm{D}$ plane that minimizes the sum of squares of deviation. The resulting projection is used as an input into the VMSI method. (c) Scatter plot between the inferred tensions using the work flow outlined and the known tensions shown in (a). The inset displays the dependence on the number of patches used to cover the sphere. As was expected, correlation is monotonic with sampling resolution.

between each patch-denoted $\lambda_{n}$. We fix such scales by defining each patch to overlap by $1 / 4$ their linear extent such that a subset of edges is involved in multiple inferred regions. Hence, the scale $\lambda_{n}$ for each patch is found by minimizing the squared difference between inferred tensions of edges shared by adjacent patches globally, subject to the constraint that the average scale is 1 to ensure a nontrivial solution, $\lambda_{n} \neq 0$.

This was used to define the patch size used in the empirical measurements during Drosophila embryogenesis. The outlined procedure was validated in silico for synthetic spherical embryos containing roughly 3000 cells, with mechanics patterned by a vertex model minimized on the surface of a sphere. An example of a simulated embryo with azimuthal pattern of tension is shown in Fig. 11(a), both in the embedding space and in the cylindrical unwrapping of the sphere, analogous to the data shown for the Drosophila embryo. As shown in Fig. 11(c), excellent agreement between the inferred and known tensions was found provided the patch size was small compared to surface curvature. The inset shows this occurred when the defined patches contained 100 or fewer cells. This was used to define the patch size used in the empirical measurements during Drosophila embryogenesis. 

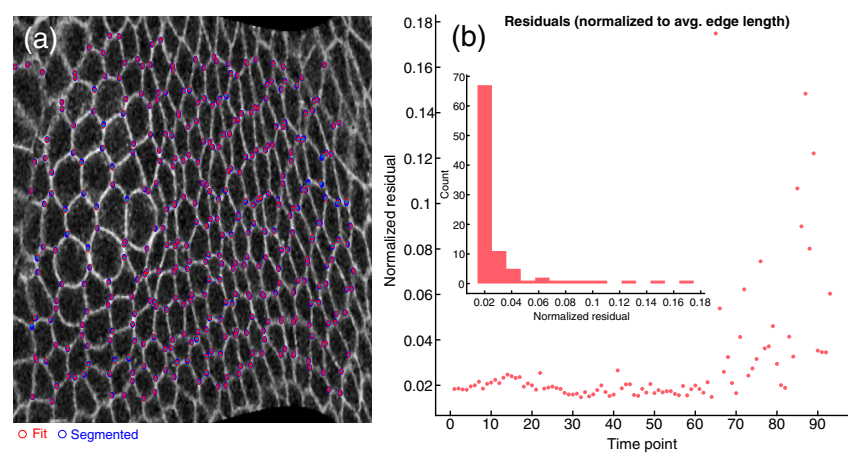

FIG. 12. (a) A confocal image of the patch of lateral ectoderm during GBE comparing vertex positions obtained by segmentation (in blue) and the position obtained by VMSI (in red). (b) The plot of residual [Eq. (5)] normalized by the average bond length over the time course of the movie analyzed. The inset displays the distribution of values. The VMSI begins to make qualitative errors during the late stage GBE, in agreement with our observation that myosin becomes unbalanced.

\section{APPENDIX F: RESIDUALS OF VMSI ALGORITHM DURING GBE}

The quality of VMSI fitted equilibrium CAP network to the observed cellular array can be obtained directly from the residual energy from minimization of Eq. (5). The minimized energy is the average squared difference (in pixels) between the segmented and fitted edges. Hence, an estimate of the overall relative error can be obtained by normalizing the final energy by the average segmented edge length in the cell array. Figure 12 compares result of image segmentation obtained by VMSI (a) and plots relative error as a function of time during the GBE process.

We note that the beginning and middle of GBE are well approximated by a CAP network in cell array, however, the relative error raises to $\sim 12 \%$ at late times, consistent with the coarse-grained myosin measurements in Fig. 5(d).

\section{APPENDIX G: DYNAMICS OF MITOTIC CELLS IN LATE GBE}

In this appendix, we discuss how we choose the time for the measurement of the long axis of premitotic cells which used in the comparison to the final mitotic axis-i.e., the measurement reported in Fig. 6(d).

Our goal was to utilize the long axis observed immediately before nuclear envelope breakdown and the subsequent apical rounding. Figure 13 displays the dynamics of both the average and standard deviation of cellular apical area (A), eccentricity (B), and the change of long 4 axis' orientation $(\mathrm{C})$ across all tracked mitotic cells.

We observed a reproducible linear increase of apical area which begins to ramp approximately $5 \mathrm{~min}$ before mitosis. Additionally, cell eccentricity was found to start decreasing at roughly the same time. As no significant drift in the orientation long axis was observed over this time frame,
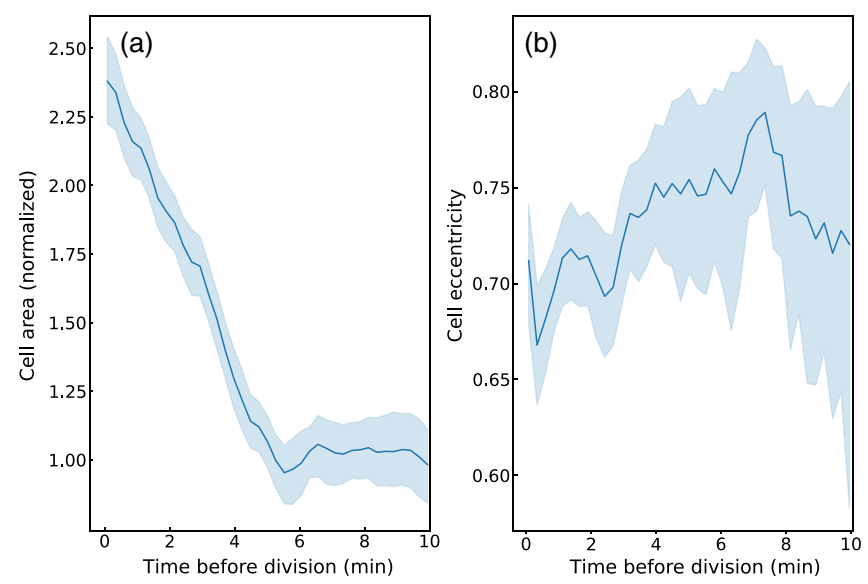

FIG. 13. Distributions of cell area (a) and cell eccentricity (b). Solid lines indicate means while surrounding outline indicates one standard deviation. We observe that the onset of the "rounding up" of the cell and the start of area growth occurs at 5 min before division.

5 min before mitosis was chosen as a reliable time to measure the premitotic long axis.

[1] R. Farhadifar, J.-C. Röper, B. Aigouy, S. Eaton, and F. Jülicher, The Influence of Cell Mechanics, Cell-Cell Interactions, and Proliferation on Epithelial Packing, Curr. Biol. 17, 2095 (2007).

[2] M. Rauzi, P. Verant, T. Lecuit, and P.-F. Lenne, Nature and Anisotropy of Cortical Forces Orienting Drosophila Tissue Morphogenesis, Nat. Cell Biol. 10, 1401 (2008).

[3] G. B. Blanchard, A. J. Kabla, N. L. Schultz, L. C. Butler, B. Sanson, N. Gorfinkiel, L. Mahadevan, and R. J. Adams, Tissue Tectonics: Morphogenetic Strain Rates, Cell Shape Change and Intercalation, Nat. Methods 6, 458 (2009).

[4] R. Etournay, M. Merkel, M. Popović, H. Brandl, N. A. Dye, B. Aigouy, G. Salbreux, S. Eaton, and F. Jülicher, TissueMiner: A Multiscale Analysis Toolkit to Quantify How Cellular Processes Create Tissue Dynamics, eLife 5, e14334 (2016).

[5] D. E. Ingber, Mechanical Control of Tissue Growth: Function Follows Form, Proc. Natl. Acad. Sci. U.S. A. 102, 11571 (2005).

[6] B. I. Shraiman, Mechanical Feedback as a Possible Regulator of Tissue Growth, Proc. Natl. Acad. Sci. U.S. A. 102, 3318 (2005).

[7] Y. Pan, I. Heemskerk, C. Ibar, B. I. Shraiman, and K. D. Irvine, Differential Growth Triggers Mechanical Feedback that Elevates Hippo Signaling, Proc. Natl. Acad. Sci. U.S.A. 113, E6974 (2016).

[8] C. Frantz, K. M. Stewart, and V. M. Weaver, The Extracellular Matrix at a Glance, J. Cell Sci. 123, 4195 (2010).

[9] A. Hartsock and W. J. Nelson, Adherens and Tight Junctions: Structure, Function and Connections to the Actin Cytoskeleton, Biochim. Biophys. Acta 1778, 660 (2008). 
[10] H. Lodish, A. Berk, C. A. Kaiser, M. Krieger, A. Bretscher, H. Ploegh, A. Amon, and K. C. Martin, Molecular Cell Biology, 8th ed. (W.H. Freeman, New York, 2016).

[11] J. M. Halbleib and W. J. Nelson, Cadherins in Development: Cell Adhesion, Sorting, and Tissue Morphogenesis, Genes Dev. 20, 3199 (2006).

[12] J. D. Franke, R. A. Montague, and D. P. Kiehart, Nonmuscle Myosin II Generates Forces That Transmit Tension and Drive Contraction in Multiple Tissues during Dorsal Closure, Curr. Biol. 15, 2208 (2005).

[13] T. Lecuit and P.-F. Lenne, Cell Surface Mechanics and the Control of Cell Shape, Tissue Patterns and Morphogenesis, Nat. Rev. Mol. Cell Biol. 8, 633 (2007).

[14] G. Salbreux, G. Charras, and E. Paluch, Actin Cortex Mechanics and Cellular Morphogenesis, Trends Cell Biol. 22, 536 (2012).

[15] M. P. Stewart, J. Helenius, Y. Toyoda, S. P. Ramanathan, D. J. Muller, and A. A. Hyman, Hydrostatic Pressure and the Actomyosin Cortex Drive Mitotic Cell Rounding, Nature (London) 469, 226 (2011).

[16] H. Honda, H. Yamanaka, and M. Dan-Sohkawa, A Computer Simulation of Geometrical Configurations during Cell Division, J. Theor. Biol. 106, 423 (1984).

[17] L. Hufnagel, A. A. Teleman, H. Rouault, S. M. Cohen, and B. I. Shraiman, On the Mechanism of Wing Size Determination in Fly Development, Proc. Natl. Acad. Sci. U.S.A. 104, 3835 (2007).

[18] N. Noll, M. Mani, I. Heemskerk, S. J. Streichan, and B. I. Shraiman, Active Tension Network Model Suggests an Exotic Mechanical State Realized in Epithelial Tissues, Nat. Phys. 13, 1221 (2017).

[19] J. M. Osborne, A. G. Fletcher, J. M. Pitt-Francis, P. K. Maini, and D.J. Gavaghan, Comparing Individual-Based Approaches to Modelling the Self-Organization of Multicellular Tissues, PLoS Comput. Biol. 13, e1005387 (2017).

[20] K. Haase and A. E. Pelling, Investigating Cell Mechanics with Atomic Force Microscopy, J. R. Soc. Interface 12, 20140970 (2015).

[21] K. Bambardekar, R. Clément, O. Blanc, C. Chardès, and P.-F. Lenne, Direct Laser Manipulation Reveals the Mechanics of Cell Contacts In Vivo, Proc. Natl. Acad. Sci. U.S.A. 112, 1416 (2015).

[22] M. S. Hutson, Y. Tokutake, M.-S. Chang, J. W. Bloor, S. Venakides, D. P. Kiehart, and G. S. Edwards, Forces for Morphogenesis Investigated with Laser Microsurgery and Quantitative Modeling, Science 300, 145 (2003).

[23] I. Bonnet, P. Marcq, F. Bosveld, L. Fetler, Y. Bellaïche, and F. Graner, Mechanical State, Material Properties and Continuous Description of an Epithelial Tissue, J. R. Soc. Interface 9, 2614 (2012).

[24] A.-L. Cost, P. Ringer, A. Chrostek-Grashoff, and C. Grashoff, How to Measure Molecular Forces in Cells: A Guide to Evaluating Genetically-Encoded FRET-Based Tension Sensors, Cell. Mol. Bioeng. 8, 96 (2015).

[25] O. Campàs, T. Mammoto, S. Hasso, R. A. Sperling, D. O'Connell, A. G. Bischof, R. Maas, D. A. Weitz, L. Mahadevan, and D. E. Ingber, Quantifying Cell-Generated Mechanical Forces within Living Embryonic Tissues, Nat. Methods 11, 183 (2014).
[26] K. Doubrovinski, M. Swan, O. Polyakov, and E. F. Wieschaus, Measurement of Cortical Elasticity in Drosophila melanogaster Embryos Using Ferrofluids, Proc. Natl. Acad. Sci. U.S.A. 114, 1051 (2017).

[27] K. Sugimura, P.-F. Lenne, and F. Graner, Measuring Forces and Stresses In Situ in Living Tissues, Development 143, 186 (2016).

[28] G. W. Brodland, V. Conte, P. G. Cranston, J. Veldhuis, S. Narasimhan, M. S. Hutson, A. Jacinto, F. Ulrich, B. Baum, and M. Miodownik, Video Force Microscopy Reveals the Mechanics of Ventral Furrow Invagination in Drosophila, Proc. Natl. Acad. Sci. U.S.A. 107, 22111 (2010).

[29] K. K. Chiou, L. Hufnagel, and B. I. Shraiman, Mechanical Stress Inference for Two Dimensional Cell Arrays, PLoS Comput. Biol. 8, e1002512 (2012).

[30] K. A. Brakke, The Surface Evolver, Exp. Math. 1, 141 (1992).

[31] S. Ishihara and K. Sugimura, Bayesian Inference of Force Dynamics during Morphogenesis, J. Theor. Biol. 313, 201 (2012).

[32] B. Guirao, S. U. Rigaud, F. Bosveld, A. Bailles, J. Lopez-Gay, S. Ishihara, K. Sugimura, F. Graner, and Y. Bellaïche, Unified Quantitative Characterization of Epithelial Tissue Development, eLife 4, e08519 (2015).

[33] V. Nier, S. Jain, C. T. Lim, S. Ishihara, B. Ladoux, and P. Marcq, Inference of Internal Stress in a Cell Monolayer, Biophys. J. 110, 1625 (2016).

[34] W. Kong, O. Loison, P. C. Shivakumar, C. Collinet, P.-F. Lenne, and R. Clément, Force Inference Predicts Local and Tissue-Scale Stress Patterns in Epithelia, https://www .biorxiv.org/content/10.1101/475012v2.

[35] G. W. Brodland, J. Veldhuis, S. Kim, M. Perrone, D. Mashburn, and M. Hutson, CellFIT: A Cellular ForceInference Toolkit Using Curvilinear Cell Boundaries, PLoS One 9 (2014).

[36] S. J. Streichan, M. F. Lefebvre, N. Noll, E. F. Wieschaus, and B. I. Shraiman, Global Morphogenetic Flow Is Accurately Predicted by the Spatial Distribution of Myosin Motors, eLife 7, e27454 (2018).

[37] B. He, K. Doubrovinski, O. Polyakov, and E. Wieschaus, Apical Constriction Drives Tissue-Scale Hydrodynamic Flow to Mediate Cell Elongation, Nature (London) 508, 392 (2014).

[38] C. Collinet, M. Rauzi, P.-F. Lenne, and T. Lecuit, Local and Tissue-Scale Forces Drive Oriented Junction Growth during Tissue Extension, Nat. Cell Biol. 17, 1247 (2015).

[39] See www.github.com/nnoll/tissueAnalysisSuite.

[40] L. D. Landau and E. M. Lifshitz, Fluid Mechanics (Pergamon Press, Oxford, 1959).

[41] H. S. M. Coxeter, Introduction to Geometry (John Wiley \& Sons, New York, 1961).

[42] C. Moukarzel, Geometrical Consequences of Foam Equilibrium, Phys. Rev. E 55, 6866 (1997).

[43] G. K. Batchelor, The Stress System in a Suspension of Force-Free Particles, J. Fluid Mech. 41, 545 (1970).

[44] L. D. Landau and E. M. Lifshitz, Course of Theoretical Physics: Theory and Elasticity (Pergamon Press, Oxford, 1986), Vol. 7.

[45] J. A. Zallen and J. T. Blankenship, Multicellular Dynamics during Epithelial Elongation, Semin. Cell Dev. Biol. 19, 263 (2008). 
[46] M. Rauzi, P.-F. Lenne, and T. Lecuit, Planar Polarized Actomyosin Contractile Flows Control Epithelial Junction Remodelling, Nature (London) 468, 1110 (2010).

[47] I. Heemskerk and S. J. Streichan, Tissue Cartography: Compressing Bio-Image Data by Dimensional Reduction, Nat. Methods 12, 1139 (2015).

[48] L. A. Baena-López, A. Baonza, and A. García-Bellido, The Orientation of Cell Divisions Determines the Shape of Drosophila Organs, Curr. Biol. 15, 1640 (2005).

[49] V. E. Foe, Mitotic Domains Reveal Early Commitment of Cells in Drosophila Embryos, Development 107, 1 (1989).

[50] S. M. da Silva and J.-P. Vincent, Oriented Cell Divisions in the Extending Germband of Drosophila, Development 134, 3049 (2007).

[51] M. Louveaux, J.-D. Julien, V. Mirabet, A. Boudaoud, and O. Hamant, Cell Division Plane Orientation Based on Tensile Stress in Arabidopsis thaliana, Proc. Natl. Acad. Sci. U.S.A. 113, E4294 (2016).

[52] A. Nestor-Bergmann, G. Goddard, and S. Woolner, Force and the Spindle: Mechanical Cues in Mitotic Spindle Orientation, in Seminars in Cell \& Developmental Biology (Elsevier, New York, 2014), Vol. 34, pp. 133-139.
[53] T. P. J. Wyatt, A. R. Harris, M. Lam, Q. Cheng, J. Bellis, A. Dimitracopoulos, A. J. Kabla, G. T. Charras, and B. Baum, Emergence of Homeostatic Epithelial Packing and Stress Dissipation through Divisions Oriented along the Long Cell Axis, Proc. Natl. Acad. Sci. U.S.A. 112, 5726 (2015).

[54] F. Bosveld, O. Markova, B. Guirao, C. Martin, Z. Wang, A. Pierre, M. Balakireva, I. Gaugue, A. Ainslie, N. Christophorou et al., Epithelial Tricellular Junctions Act as Interphase Cell Shape Sensors to Orient Mitosis, Nature (London) 530, 495 (2016).

[55] U. Krzic, S. Gunther, T. E. Saunders, S. J. Streichan, and L. Hufnagel, Multiview Light-Sheet Microscope for Rapid In Toto Imaging, Nat. Methods 9, 730 (2012).

[56] A. D. Edelstein, M. A. Tsuchida, N. Amodaj, H. Pinkard, R. D. Vale, and N. Stuurman, Advanced Methods of Microscope Control Using Manager Software, J. Biol. Methods 1, e10 (2014).

[57] S. Preibisch, F. Amat, E. Stamataki, M. Sarov, R. H. Singer, E. Myers, and P. Tomancak, Efficient Bayesian-Based Multiview Deconvolution, Nat. Methods 11, 645 (2014).

[58] S. W. Walker, FELICITY: A Matlab/C++ Toolbox for Developing Finite Element Methods and Simulation Modeling, SIAM J. Sci. Comput. 40, C234 (2018). 